\title{
Exponential convergence of Gauss-- Jacobi quadratures for singular integrals over simplices in arbitrary dimension
}

Article

Published Version

Chernov, A. and Schwab, C. (2012) Exponential convergence of Gauss--Jacobi quadratures for singular integrals over simplices in arbitrary dimension. SIAM Journal on Numerical Analysis (SINUM), 50 (3). pp. 1433-1455. ISSN 0036-1429 doi: https://doi.org/10.1137/100812574 Available at https://centaur.reading.ac.uk/33216/

It is advisable to refer to the publisher's version if you intend to cite from the work. See Guidance on citing.

To link to this article DOI: http://dx.doi.org/10.1137/100812574

Publisher: Society for Industrial and Applied Mathematics

Publisher statement: (c 2012, Society for Industrial and Applied Mathematics

All outputs in CentAUR are protected by Intellectual Property Rights law, including copyright law. Copyright and IPR is retained by the creators or other copyright holders. Terms and conditions for use of this material are defined in the End User Agreement.

www.reading.ac.uk/centaur 
Central Archive at the University of Reading

Reading's research outputs online 


\title{
EXPONENTIAL CONVERGENCE OF GAUSS-JACOBI QUADRATURES FOR SINGULAR INTEGRALS OVER SIMPLICES IN ARBITRARY DIMENSION*
}

\author{
ALEXEY CHERNOV ${ }^{\dagger}$ AND CHRISTOPH SCHWAB
}

\begin{abstract}
Galerkin discretizations of integral operators in $\mathbb{R}^{d}$ require the evaluation of integrals $\int_{S(1)} \int_{S(2)} f(x, y) d y d x$, where $S^{(1)}, S^{(2)}$ are $d$-dimensional simplices and $f$ has a singularity at $x=y$. In [A. Chernov, T. von Petersdorff, and C. Schwab, M2AN Math. Model. Numer. Anal., 45 (2011), pp. 387-422] we constructed a family of $h p$-quadrature rules $Q_{N}$ with $N$ function evaluations for a class of integrands $f$ allowing for algebraic singularities at $x=y$, possibly nonintegrable with respect to either $d x$ or $d y$ (hypersingular kernels) and Gevrey- $\delta$ smooth for $x \neq y$. This is satisfied for kernels from broad classes of pseudodifferential operators. We proved that $Q_{N}$ achieves the exponential convergence rate $\mathcal{O}\left(\exp \left(-r N^{\gamma}\right)\right)$ with the exponent $\gamma=1 /(2 d \delta+1)$. In this paper we consider a special singularity $\|x-y\|^{\alpha}$ with real $\alpha$ which appears frequently in appplication and prove that an improved convergence rate with $\gamma=1 /(2 d \delta)$ is achieved if a certain one-dimensional Gauss-Jacobi quadrature rule is used in the (univariate) "singular coordinate." We also analyze approximation by tensor Gauss-Jacobi quadratures in the "regular coordinates." We illustrate the performance of the new Gauss-Jacobi rules on several numerical examples and compare it to the hp-quadratures from [A. Chernov, T. von Petersdorff, and C. Schwab, M2AN Math. Model. Numer. Anal., 45 (2011), pp. 387-422].
\end{abstract}

Key words. Gauss-Jacobi quadrature, numerical integration, high dimensional integrands, hypersingular integrals, integral equations, Gevrey regularity, exponential convergence

AMS subject classifications. 65N30, 65D30, 32A55

DOI. $10.1137 / 100812574$

1. Introduction and notation. A basic problem in the numerical analysis of Galerkin discretizations of singular integral equations involves computation of double integrals of the type

$$
\int_{x \in \Omega_{\mathrm{op}}} \int_{y \in \Omega_{\mathrm{op}}} K(x, y) \phi_{j}(y) \phi_{i}(x) d y d x .
$$

Here, the set $\Omega_{\mathrm{op}}$ denotes the domain of definition of the pseudodifferential operator with Schwartz kernel $K(x, y)$. Typically, the kernel $K(x, y)$ is smooth for $x \neq y$ but becomes strongly singular at $x=y[6,13]$. In this case, integration with respect to $K(x, y)$ must be interpreted in a suitable sense; cf., e.g., [9]. In finite element type Galerkin discretizations, the functions $\phi_{j}$ are piecewise polynomials on closed simplices. Then, this amounts to computing integrals of the type

$$
I=\int_{x \in S^{(1)}} \int_{y \in S^{(2)}} K(x, y) \phi_{j}(y) \phi_{i}(x) d y d x,
$$

where $S^{(1)}, S^{(2)}$ are closed simplices of the mesh and the restrictions $\left.\phi_{i}\right|_{S^{(1)}},\left.\phi_{j}\right|_{S^{(2)}}$ are smooth functions in $S^{(1)}, S^{(2)}$. If the simplices touch or coincide, the integrand is singular. Throughout, we work under an assumption of shape regularity of $S^{(1)}, S^{(2)}$.

\footnotetext{
${ }^{*}$ Received by the editors October 22, 2010; accepted for publication (in revised form) January 23, 2012; published electronically June 5, 2012.

http://www.siam.org/journals/sinum/50-3/81257.html

${ }^{\dagger}$ Hausdorff Center for Mathematics and Institute for Numerical Simulation, University of Bonn, 53115 Bonn, Germany (chernov@hcm.uni-bonn.de).

${ }^{\ddagger}$ Seminar für Angewandte Mathematik, ETH Zürich, 8092 Zürich, Switzerland (schwab@math. ethz.ch). This author's work was supported by ERC AdG 247277.
} 
Assumption 1. For closed simplices $S^{(1)}, S^{(2)}$ in $\mathbb{R}^{d}$ let $h_{j}:=\operatorname{diam} S^{(j)}$ denote the diameters, and let $\rho_{j}$ denote the radii of the largest ball contained in $S^{(j)}$ for $j=1,2$. The simplices $S^{(1)}, S^{(2)}$ are shape regular in the following sense: There exist $C_{1}, C_{2}>0$ with

$$
\frac{h_{j}}{\rho_{j}} \leq C_{1}
$$

If $S^{(1)} \cap S^{(2)}$ is empty, then we let $k:=-1$ and assume

$$
\operatorname{dist}\left(S^{(1)}, S^{(2)}\right) \geq C_{2}^{-1} \max \left\{h_{1}, h_{2}\right\} .
$$

If $S^{(1)} \cap S^{(2)}$ is nonempty, the intersection is the convex hull of $k+1$ vertices of $S^{(j)}$ for $j=1,2$ with $k \in\{0, \ldots, d\}$. We assume that $S^{(1)}, S^{(2)}$ are nondegenerate in the sense that the matrices $A^{(j)}, B^{(j)}$ defined in Step 1 below are uniformly nondegenerate.

These assumptions are satisfied if $S^{(1)}, S^{(2)}$ are part of a shape regular finite element mesh. Note that this condition is preserved under regular mesh refinements. The convergence estimate of the type (1.6) below will hold for any values $C_{j}$, but the constants will deteriorate for large values of $C_{j}$.

If the original domain of definition $\Omega_{\mathrm{op}}$ is curved or consists of a piecewise analytic or Gevrey- $\delta$ regular manifold in a higher dimensional space (e.g., the boundary of a polyhedron in $\left.\mathbb{R}^{d+1}\right)$, the functions $\left.\phi_{i}\right|_{S^{(1)}}$ and $\left.\phi_{j}\right|_{S^{(2)}}$ might include parametric mappings, Jacobians, and cutoff functions but are still Gevrey- $\delta$ regular functions on $S^{(1)}$ and $S^{(2)}$ (cf. Proposition A.2 in the appendix) in the following sense.

Definition 1.1. Let $\Omega \subset \mathbb{R}^{m}$ be a closed bounded set, and let $\delta \geq 1$. A function $f: \Omega \rightarrow \mathbb{R}$ is Gevrey- $\delta$ regular $f \in G^{\delta}(\Omega)$ iff there exist $A_{0}, A_{1}>0$ so that

$$
\left|D^{\nu} f(x)\right| \leq A_{0} A_{1}^{|\nu|}(\nu !)^{\delta} \quad \forall x \in \Omega, \quad \forall \nu \in \mathbb{N}_{0}^{m} .
$$

$A$ vector-valued $f \in G^{\delta}(\Omega)$ if (1.2) holds for every component of $f$.

We remark that for $\delta=1$, Gevrey regularity implies analyticity. The case $\delta>1$ corresponds to nonanalytic but smooth classes of functions. We refer the reader to $[1,3]$ for more details on Gevrey classes $G^{\delta}(\Omega)$ for $\delta>1$.

In all these cases the integrand can be written as a function $F(x, y, y-x) \in$ $L^{1}\left(S^{(1)} \times S^{(2)}\right)$, where $F(x, y, z)$ is Gevrey- $\delta$ regular in $x, y, z$ for $z \neq 0$ but might have a singularity at $z=0$. Precisely, the integral $I$ from (1.1) takes the form

$$
I=\int_{x \in S^{(1)}} \int_{y \in S^{(2)}} F(x, y, y-x) d y d x,
$$

and $F$ satisfies the following assumption.

Assumption 2. There exist $\alpha>k-2 d, A_{0}, A_{1}>0$, and $\delta \geq 1$ so that for all $\nu=\left(\nu_{x}, \nu_{y}, \nu_{z}\right) \in \mathbb{N}_{0}^{3 d}, x \in S^{(1)}, y \in S^{(2)}$, and $z \in S^{(2)}-S^{(1)}$,

$$
\left|D^{\nu} F(x, y, z)\right| \leq A_{0} A_{1}^{|\nu|}(\nu !)^{\delta}\|z\|^{\min \left(\alpha-\left|\nu_{z}\right|, 0\right)} .
$$

The parameter $\alpha$ is termed the order of singularity.

Our aim is to approximate the singular integral $I$ from (1.3) by a quadrature rule,

$$
Q_{N}=\sum_{\lambda=1}^{N} F\left(x_{\lambda}, y_{\lambda}, z_{\lambda}\right) w_{\lambda},
$$

Copyright $@$ by SIAM. Unauthorized reproduction of this article is prohibited. 
with the quadrature nodes $\left(x_{\lambda}, y_{\lambda}, z_{\lambda}\right) \in S^{(1)} \times S^{(2)} \times\left(S^{(2)}-S^{(1)}\right) \backslash\{0\}$ and positive quadrature weights $w_{\lambda}$.

Remark 1. In exact arithmetic $z_{\lambda}=y_{\lambda}-x_{\lambda}$, and the intermediate storage of $z_{\lambda}$ is redundant in an implementation. In the finite precision, float point arithmetic, evaluation of the difference $y_{\lambda}-x_{\lambda}$ in general is susceptive to subtractive cancellation. This effect was pointed out in [3, Remark 3.7] and is illustrated on a numerical example in [3, Figure 5]. To avoid such subtractive cancellation, we propose keeping $z_{\lambda}$ and using transformations avoiding subtractive cancellation. This is the reason for (3.15).

In [3] we constructed a family of $h p$-quadrature (or "variable-order, composite") rules $Q_{N}$ of the form (1.5) and proved the exponential convergence with respect to $N$ :

$$
\left|I-Q_{N}\right| \leq C \exp \left(-r N^{\gamma}\right)
$$

with $r>0$ independent of $N$ and $d$ and the exponent $\gamma=1 /(2 d \delta+1)$. An improved convergence rate with $\gamma=1 /(2 d \delta)$ is achieved if $k=-1$ (no singularity) or when $\alpha>$ $k-2 d$ is an integer. Our construction consists of a sequence of analytic, regularizing coordinate transforms which reduce the dimensionality of the singularity and, at the same time, simplify the domain of integration. In particular, we prove here that after this parameter transform the domain of integration is a cube $[0,1]^{2 d}$ and the singular support of the integrand is univariate while the integrand is Gevrey- $\delta$ regular in the remaining $2 d-1$ coordinates with the same Gevrey exponent $\delta$.

In this paper we prove that the improved convergence rate (1.6) with $\gamma=1 /(2 d \delta)$ can be achieved for a particular important class of integrands involving kernels of the type

$$
F(x, y, z)=\|z\|^{\alpha} \tilde{F}(x, y, z) .
$$

Here $\alpha>k-2 d$ is a real number and $\tilde{F} \in G^{\delta}\left(S^{(1)} \times S^{(2)} \times\left(S^{(2)}-S^{(1)}\right)\right)$ in the sense of Definition 1.1. In other words, this means that under assumption (1.7) our quadrature rule for singular integrands achieves asymptotically the same precision as a tensor Gauss-Legendre or Gauss-Jacobi quadrature rule for a Gevrey- $\delta$ integrand $\tilde{F}$ without singularity using the same number of function evaluations. This result has been known for integer $\alpha$ in dimension $d=1,2$ and for $\delta=1$, which corresponds to layer or volume potentials related to elliptic PDEs; see Remark 4 and, e.g., [10, Chapter 5], [12]. Theorem 5.4 below extends this result to the case of general $d \geq 1$ and noninteger $\alpha$. The main ingredient for this convergence result is Theorem 4.1, showing that for an integrand $F$ satisfying (1.7), the transformed integrand has a singularity of the form $t_{1}^{\tilde{\alpha}}$ with $\tilde{\alpha}=\alpha+2 d-k-1>-1$. This can be treated as a weight of a one-dimensional Gauss-Jacobi quadrature rule on the interval $t_{1} \in[0,1]$. Clearly, in order to take advantage of this property, the singularity order $\alpha$ must be known, which was not required in [3] but is usually the case in applications.

The paper is organized as follows. In section 2 we introduce three basic classes of parametrizations of the reference simplex $S_{d}$ which will be frequently used later on. In section 3 we describe Steps 1-8 of our coordinate transformations. Section 4 is dedicated to the detailed regularity analysis for integrands of the type (1.7). The convergence proofs justifying the improved convergence rates for the Gauss-Jacobi quadrature rules are given in section 5 . In section 6 we give several numerical examples illustrating the improved convergence of the new Gauss-Jacobi $2 d$-dimensional quadrature rules and compare it to the $h p$-quadratures from [3]. The appendix contains two technical propositions on the regularity of Gevrey- $\delta$ functions. 
2. Basic parametrizations of the reference simplex. We adopt notation from [3]. Consider a subset $N=\left\{n_{1}, \ldots, n_{k}\right\}$ of $\{1, \ldots, d\}$ with $n_{1}<\cdots<n_{k}$. For any $x \in \mathbb{R}^{d}$ we denote $x_{N}:=\left(x_{n_{1}}, \ldots, x_{n_{k}}\right) \in \mathbb{R}^{k}$ and $x^{(N)} \in \mathbb{R}^{d}$ by $x_{j}^{(N)}:=x_{j}$ for $j \in N, x_{j}^{(N)}:=0$ for $j \notin N$. We write $x \geq 0$ for a vector $x$ iff $x_{j} \geq 0$ for all $j$. For a finite set $N$ we denote by $\# N$ the number of its elements. For $x \in \mathbb{R}^{m}$ we use the notation

$$
\sigma_{\left(x_{1}, \ldots, x_{m}\right)}=\sigma_{x}:= \begin{cases}1-\sum_{j=1}^{m} x_{j}, & m \geq 1 \\ 1, & m=0\end{cases}
$$

In this section we consider three basic analytic parametrizations of the reference simplex defined by

$$
S_{d}:=\left\{\left(x_{1}, \ldots, x_{d}\right) \mid x \geq 0, x_{1}+\cdots+x_{d} \leq 1\right\}
$$

and the associate transformations of the integral over $S_{d}$

$$
Y:=\int_{x \in S_{d}} \varphi(x) d x
$$

for some $\varphi$ being integrable in $S_{d}$. The associated degenerate coordinate transforms will be frequently used in the regularizing coordinate transforms in section 3 .

2.1. First parametrization of $x \in S_{d}$ by $(t, y) \in S_{d-k} \times S_{k}$. Definition (2.2) directly yields the parametrization

$$
\begin{aligned}
S_{d} & =\left\{x \mid 0 \leq x_{j} \leq \sigma_{\left(x_{j+1}, \ldots, x_{d}\right)}, 1 \leq j \leq d\right\} \\
& =\left\{x \mid\left(x_{j}, \ldots, x_{d}\right) \in S_{d-j+1}, 1 \leq j \leq d\right\}
\end{aligned}
$$

Let us fix some $k \in\{0, \ldots, d\}$ and introduce the new variables $t_{j}, y_{j}$ by recursion over the dimension:

$$
t_{j}:=x_{k+j}, \quad j=1, \ldots, d-k, \quad \text { and } \quad y_{j}:=\frac{x_{j}}{\sigma_{\left(x_{k+1}, \ldots, x_{d}\right)}}=\frac{x_{j}}{\sigma_{t}}, \quad j=1, \ldots, k .
$$

Then for $j=1, \ldots, k$ there holds

$$
\begin{aligned}
0 \leq y_{j}=\frac{x_{j}}{\sigma_{t}} \leq \frac{1-x_{j+1}-\cdots-x_{d}}{\sigma_{t}}=1-\frac{x_{j+1}+\cdots+x_{k}}{\sigma_{t}} & =1-y_{j+1}-\cdots-y_{k} \\
& =\sigma_{\left(y_{j+1}, \ldots, y_{k}\right)}
\end{aligned}
$$

hence $y \in S_{k}$ and $t \in S_{d-k}$ due to (2.4). This yields the parametrization

$$
S_{d}=\left\{\left(\sigma_{t} y, t\right) \mid t \in S_{d-k}, y \in S_{k}\right\} .
$$

Inserting this parametrization into (2.3), we obtain

$$
Y=\int_{t \in S_{d-k}} \int_{y \in S_{k}} \varphi\left(\sigma_{t} y, t\right) \sigma_{t}^{k} d y d t
$$

Copyright (C) by SIAM. Unauthorized reproduction of this article is prohibited. 

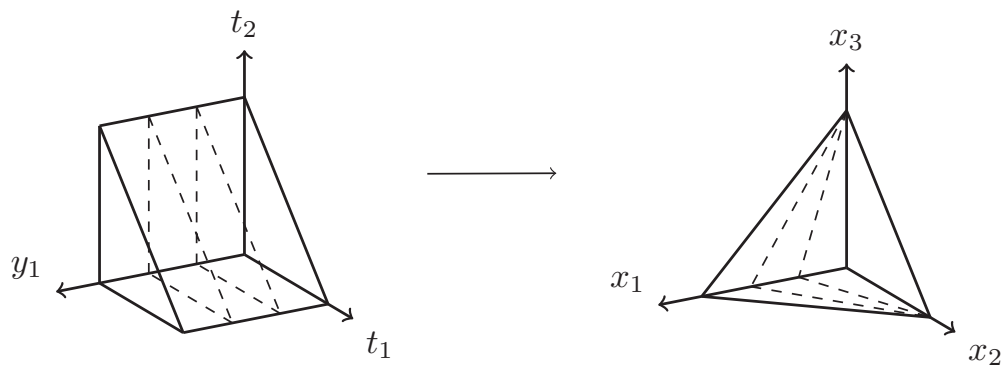

FIG. 2.1. First parametrization (2.5) for $d=3$ and $k=1$.
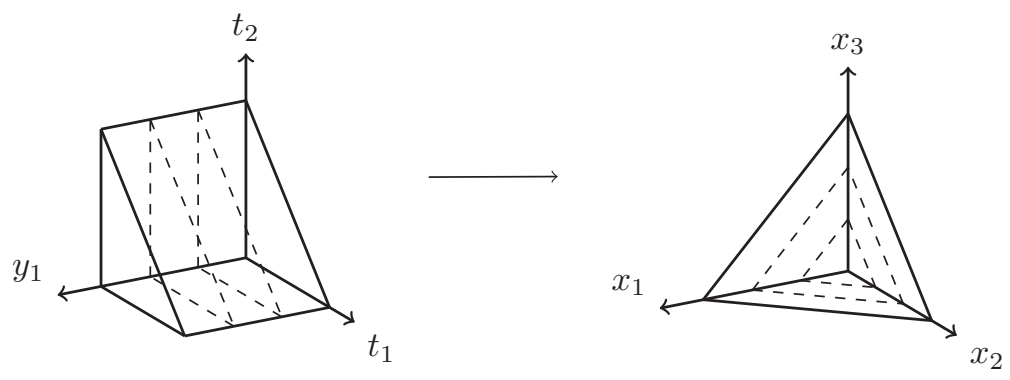

FIG. 2.2. Second parametrization (2.9) for $d=3$.

2.2. Second parametrization of $x \in S_{d}$ by $(t, y) \in S_{d-1} \times[0,1]$. Recall definition (2.2) of $S_{d}$. Then

$$
B_{d}:=\left\{\left(x_{1}, \ldots, x_{d}\right) \mid x \geq 0, x_{1}+\cdots+x_{d}=1\right\}
$$

is the face of $S_{d}$ which does not include the origin $x=0$. Basic calculations give alternative parametrizations of $B_{d}$ and $S_{d}$ :

$$
B_{d}=\left\{\left(\sigma_{t}, t\right) \mid t \in S_{d-1}\right\}, \quad S_{d}=\left\{y\left(x_{1}, \ldots, x_{d}\right) \mid x \in B_{d}, y \in[0,1]\right\} .
$$

Their combination yields

$$
S_{d}=\left\{\left(\sigma_{t} y, y t\right) \mid t \in S_{d-1}, y \in[0,1]\right\}
$$

and, upon inserting this into (2.3),

$$
Y=\int_{t \in S_{d-1}} \int_{y \in[0,1]} \varphi\left(\sigma_{t} y, y t\right) y^{d-1} d y d t
$$

Note that (2.5) for $k=1$ and (2.9) are two different parametrizations of $S_{d}$ by $S_{d-1} \times[0,1]$; see Figures 2.1 and 2.2 .

2.3. Third parametrization of $x \in S_{d}$ by $t \in[0,1]^{d}$. We introduce the following parametrization componentwise:

$$
x_{j}=\chi_{j}(t):= \begin{cases}t_{1} \ldots t_{j}\left(1-t_{j+1}\right), & j=1, \ldots, d-1, \\ t_{1} \ldots t_{d} & j=d .\end{cases}
$$

Copyright $@$ by SIAM. Unauthorized reproduction of this article is prohibited. 


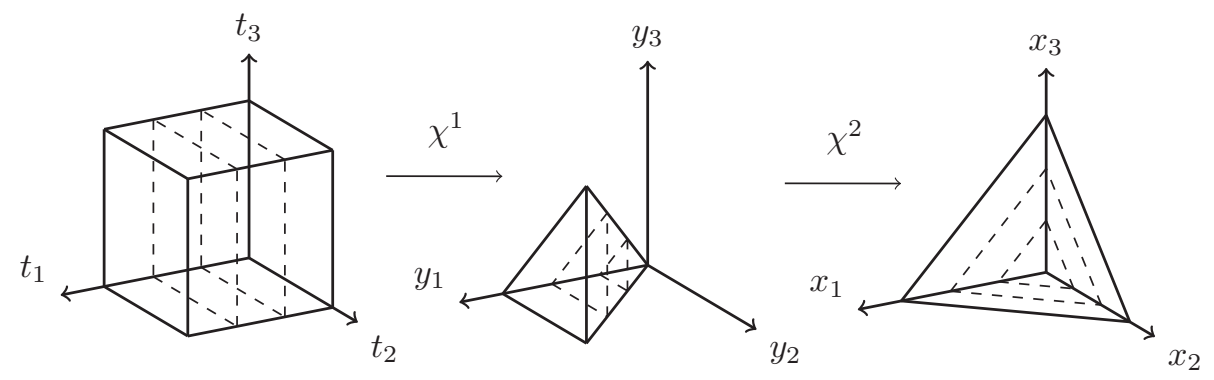

FIG. 2.3. Third parametrization (2.12) for $d=3$.

We denote $\chi(t):=\left(\chi_{1}(t), \ldots, \chi_{d}(t)\right)$. For an arbitrary $t \in[0,1]^{d}$ we obtain $0 \leq$ $\sum_{j=1}^{d} \chi_{j}(t)=t_{1} \leq 1$. This yields the parametrization

$$
S_{d}=\left\{x=\chi(t) \mid t \in[0,1]^{d}\right\} .
$$

We claim that the integral in (2.3) equals

$$
Y=\int_{t \in[0,1]^{d}} \varphi(\chi(t)) J_{\chi}(t) d t, \quad J_{\chi}(t)=t_{1}^{d-1} t_{2}^{d-2} \ldots t_{d-1}
$$

In order to show (2.13) we split $\chi=\chi^{2} \circ \chi^{1}$, where

$$
\chi_{j}^{1}(t):=t_{1} \ldots t_{j}, \quad 1 \leq j \leq d, \quad \chi_{j}^{2}(y):=\left\{\begin{array}{cc}
y_{j}-y_{j+1}, & 1 \leq j \leq d-1, \\
y_{d}, & j=d
\end{array}\right.
$$

see Figure 2.3. Then

$$
J_{\chi}(t)=\left|\operatorname{det}\left(\frac{\partial \chi}{\partial t}\right)\right|=\left|\operatorname{det}\left(\frac{\partial \chi^{1}}{\partial t}\right)\right|=t_{1}^{d-1} t_{2}^{d-2} \ldots t_{d-1}
$$

and (2.13) holds true.

3. Coordinate transformations. In this section we revisit coordinate transformations from [3], allowing us to rewrite (1.3) as an integral over a unit cube $[0,1]^{2 d}$. Here, we introduce slightly different notation which is more convenient for the integrand functions under consideration here. In particular, we explicitly give a sequence of coordinate transforms $\Phi_{\varkappa}$ which allows us to rewrite (1.3) as an integral over a unit cube $[0,1]^{2 d}$ :

$$
I=\int_{S^{(1)}} \int_{S^{(2)}} F(x, y, y-x) d y d x=\int_{t \in[0,1]^{2 d}} \sum_{\varkappa=1}^{K}\left(F \circ \Phi_{\varkappa}\right)(t) J_{\Phi_{\varkappa}}(t) d t .
$$

More importantly, $\Phi_{\varkappa}$ isolates the singularity in the univariate direction $t_{1}$. Then, $I$ in (3.1) is approximated numerically on the unit cube by a suitable quadrature rule with nodes $\left\{t_{\mu}\right\}_{\mu=1}^{n}$ and weights $\left\{\eta_{\mu}\right\}_{\mu=1}^{n}$ (a specific one-dimensional quadrature rule must be applied in the "singular" direction $t_{1}$ ), leading to the quadrature rule in the physical domain

$$
Q_{N}=\sum_{\mu=1}^{n} \sum_{\varkappa=1}^{K} F \circ \Phi_{\varkappa}\left(t_{\mu}\right) J_{\Phi_{\varkappa}}\left(t_{\mu}\right) \eta_{\mu} \equiv \sum_{\mu=1}^{n} \sum_{\varkappa=1}^{K} F\left(x_{\mu \varkappa}, y_{\mu \varkappa}, z_{\mu \varkappa}\right) w_{\mu \varkappa},
$$

with the total number of nodes $N=n K$. The transformed nodes and weights are 
given in terms of the backward transform

$$
\left(x_{\mu \varkappa}, y_{\mu \varkappa}, z_{\mu \varkappa}\right):=\Phi_{\varkappa}\left(t_{\mu}\right), \quad w_{\mu \varkappa}:=J_{\Phi_{\varkappa}}\left(t_{\mu}\right) \eta_{\mu} .
$$

Enumeration with the single index $(\mu, \kappa) \rightarrow \lambda$ gives the desired quadrature rule (1.5). Hence, to obtain a quadrature on the physical domain $S^{(1)} \times S^{(2)}$, one needs to evaluate $\Phi_{\varkappa}(t)$ and $J_{\Phi_{\varkappa}}(t)$ for any $t \in[0,1]^{2 d}$. Explicit evaluation of $\Phi_{\varkappa}$ and $J_{\Phi_{\varkappa}}$ is the focus of this section. To this end it will be convenient to split the evaluation into eight steps, which we discuss next.

Step 1: Transform $(x, y) \in S^{(1)} \times S^{(2)}$ to $(u, v) \in S_{d} \times S_{d}$. Suppose that $S^{(j)}$ are given by their vertices $v^{(j, 0)}, \ldots, v^{(j, d)}$ such that $v^{(1, j)}=v^{(2, j)}$ for $j=0, \ldots, k$ and $v^{(1, j)} \neq v^{(2, j)}$ for $j=k+1, \ldots, d$. We agree that values $k \in\{-1,0,1, \ldots, d\}$ are allowed. Then the case $k=-1$ represents two disjoint simplices, and $k=-1,0,1, \ldots d$ represents the dimension of $S^{(1)} \cap S^{(2)}$ (with the convention that the dimension of the empty set equals -1 ).

From now on we agree that all vectors in $\mathbb{R}^{d}$ are given by the row vectors of their components. Consider two $d \times d$ transformation matrices

$$
A^{(j)}:=\left(\begin{array}{c}
v^{(j, 1)}-v^{(j, 0)} \\
\cdots \\
v^{(j, d)}-v^{(j, 0)}
\end{array}\right), \quad j=1,2 .
$$

They allow us to parametrize $x \in S^{(1)}, y \in S^{(2)}$ by $u, v \in S_{d}$ via the affine transform

$$
x=v^{(1,0)}+u A^{(1)}, \quad y=v^{(2,0)}+v A^{(2)} .
$$

In the case of nontrivial intersection $(0 \leq k \leq d)$ we observe that $v^{(2,0)}=v^{(1,0)}$ and the first $k$ rows of $A^{(1)}$ and $A^{(2)}$ coincide:

$$
A^{(j)}=\left(\begin{array}{l}
B \\
B^{(j)}
\end{array}\right), \quad B:=\left(\begin{array}{c}
v^{(j, 1)}-v^{(j, 0)} \\
\cdots \\
v^{(j, k)}-v^{(j, 0)}
\end{array}\right), \quad B^{(j)}:=\left(\begin{array}{c}
v^{(j, k+1)}-v^{(j, 0)} \\
\cdots \\
v^{(j, d)}-v^{(j, 0)}
\end{array}\right),
$$

where $B \in \mathbb{R}^{k \times d}$ and $B^{(j)} \in \mathbb{R}^{(d-k) \times d}$. Let $\hat{u}:=\left(u_{1}, \ldots, u_{k}\right), \check{u}:=\left(u_{k+1}, \ldots, u_{d}\right)$, and similarly for $v$; then for $\hat{z}:=\hat{v}-\hat{u}$ and

$$
z=y-x=\hat{z} B+\check{v} B^{(2)}-\check{u} B^{(1)}
$$

there holds

$$
\begin{aligned}
I & =\int_{u \in S_{d}} \int_{v \in S_{d}} G(u, v, \hat{v}-\hat{u}) d v d u \quad \text { with } \\
G(u, v, \hat{z}) & :=\tilde{J} \cdot F\left(v^{(1,0)}+u A^{(1)}, v^{(2,0)}+v A^{(2)}, \hat{z} B+\check{v} B^{(2)}-\check{u} B^{(1)}\right), \\
\tilde{J} & =\left|\operatorname{det} A^{(1)} \operatorname{det} A^{(2)}\right| .
\end{aligned}
$$

The integrand $G$ is singular if $(\check{u}, \check{v}, \hat{z})=0$; cf. $[3,(3.11)]$ and part (i) of the proof of Theorem 4.1 below.

In the case $k=0$ we adopt the convention $\check{u}:=u, \check{v}:=v$, and $\hat{u}, \hat{v}, \hat{z}$ do not appear in the integrand functions. There holds $A^{(j)} \equiv B^{(j)}$, and the integral (3.6) takes the form

$$
\begin{aligned}
I & =\int_{u \in S_{d}} \int_{v \in S_{d}} G(u, v) d v d u \quad \text { with } \\
G(u, v) & :=\tilde{J} \cdot F\left(v^{(1,0)}+u A^{(1)}, v^{(2,0)}+v A^{(2)}, v A^{(2)}-u A^{(1)}\right) .
\end{aligned}
$$

Copyright $@$ by SIAM. Unauthorized reproduction of this article is prohibited. 
In the case of disjoint simplices $(k=-1)$ we have $\check{u}:=u, \check{v}:=v$, and

$$
z=y-x=v^{(2,0)}-v^{(1,0)}+v A^{(2)}-u A^{(1)} .
$$

In this case (3.8) also holds with the integrand

$$
G(u, v):=\tilde{J} \cdot F\left(v^{(1,0)}+u A^{(1)}, v^{(2,0)}+v A^{(2)}, v^{(2,0)}-v^{(1,0)}+v A^{(2)}-u A^{(1)}\right) .
$$

Step 2 for $k=-1$ : Transform $(u, v) \in S_{d} \times S_{d}$ to $(u, v) \in[0,1]^{2 d}$. In case $k=-1$ the resulting integrand $G$ in (3.11) is Gevrey- $\delta$ regular (or analytic if $\delta=1)$ in $S_{d} \times S_{d}$. We parametrize $S_{d}$ by $[0,1]^{d}$ as in (2.12) and obtain

$$
I=\int_{(\bar{u}, \bar{v}) \in[0,1]^{2 d}} G(\chi(\bar{u}), \chi(\bar{v})) \bar{u}_{1}^{d-1} \ldots \bar{u}_{d-1} \bar{v}_{1}^{d-1} \ldots \bar{v}_{d-1} d \bar{v} d \bar{u} .
$$

According to [3, Proposition 3.3], the tensor product Gauss-Legendre quadrature rule achieves an exponential rate of convergence of the type (1.6) with $\gamma=1 /(2 d \delta)$ for such integrands. In Theorem 5.4 below we prove that the Gauss-Jacobi quadrature rule absorbing monomial weights in (3.12) converges asymptotically at the same rate as the Gauss-Legendre quadrature rule.

The remaining coordinate transformations in Steps 2-8 are relevant only in the case $0 \leq k \leq d$.

Step 2: Transform $(u, v) \in S_{d} \times S_{d}$ to $(\check{u}, \check{v}, \tilde{v}, \tilde{u}) \in S_{d-k} \times S_{d-k} \times S_{k} \times S_{k}$. If $k \geq 1$, the intersection $S^{(1)} \cap S^{(2)}$ has a positive dimension $k$. In this case we will transform the integration over the intersection in Steps 2-6. If $k=0$, the simplex $S_{k}$ degenerates. In this case we say that $S_{0}$ is zero-dimensional. Similarly, we say that $\tilde{u} \in S_{0}$ is a zero-dimensional variable. We will use this terminology throughout the paper.

In the case $k=0$ we can skip Steps $2-6$ and go directly to Step 7 below because the intersection $S^{(1)} \cap S^{(2)}$ degenerates to a point. According to (2.5) we have

$$
S_{d}=\left\{\left(\sigma_{\check{u}} \tilde{u}, \check{u}\right) \mid \tilde{u} \in S_{k}, \check{u} \in S_{d-k}\right\}
$$

and similarly for $v$; thus it follows that

$$
I=\int_{\check{u} \in S_{d-k}} \int_{\check{v} \in S_{d-k}} \int_{\tilde{v} \in S_{k}} \int_{\tilde{u} \in S_{k}} H(\check{u}, \check{v}, \tilde{v}, \tilde{u}) d \tilde{u} d \tilde{v} d \check{v} d \check{u}
$$

(cf. (2.6)), where

$$
H(\check{u}, \check{v}, \tilde{v}, \tilde{u}):=G\left(\sigma_{\check{u}} \tilde{u}, \check{u}, \sigma_{\check{v}} \tilde{v}, \check{v}, \sigma_{\check{v}} \tilde{v}-\sigma_{\check{u}} \tilde{u}\right) \sigma_{\check{u}}^{k} \sigma_{\tilde{v}}^{k} .
$$

The integrand $H$ is singular at $(\check{u}, \check{v}, \tilde{v}-\tilde{u})=0$; see [3, Lemma 4.12] and part (ii) of the proof of Theorem 4.1.

Accurate float point evaluation of $\hat{z}=\sigma_{\tilde{v}} \tilde{v}-\sigma_{\tilde{u}} \tilde{u}$ is important for preventing subtractive cancellation of digits; cf. [3, Remark 3.7]. In particular, evaluation of $\hat{z}$ in the form

$$
\hat{z}=\sigma_{\check{v}} \tilde{z}+\tilde{u} \sum_{i=1}^{d-k}\left(\check{u}_{i}-\check{v}_{i}\right),
$$

where $\tilde{z}=\tilde{v}-\tilde{u}$ (see Step 3), helps to avoid subtractive cancellation. 
This evaluation is unfortunately not possible if $\Omega_{\mathrm{op}}$ is a piecewise smooth manifold in a higher dimensional space. A possible solution in this case is to use an asymptotic expansion of the integral kernel. We do not elaborate on the details and instead refer the reader to [7, Chapter 7]. In particular, we refer the reader to [7, section 7.2] for the change of coordinates in pseudohomogeneous kernels and to [7, section 8.5] for surface potentials on a boundary manifold.

We mention that $S_{k}$ is zero-dimensional if $k=0$. In this case $\check{u} \equiv u, \check{v} \equiv v$, and (3.13) formally hold in view of (3.8), and (3.9) is reduced to

$$
I=\int_{\check{u} \in S_{d}} \int_{\check{v} \in S_{d}} H(\check{u}, \check{v}) d \check{v} d \check{u} \quad \text { with } \quad H(\check{u}, \check{v}):=G(u, v) .
$$

Step 3: Transform $(\tilde{\boldsymbol{v}}, \tilde{u}) \in S_{k} \times S_{k}$ to $\left\{(\tilde{z}, \tilde{u}) \mid \tilde{z} \in A_{k}, \tilde{u} \in E_{k}(\tilde{z})\right\}$. Let $\tilde{z}:=\tilde{v}-\tilde{u} \in A_{k}$ where $A_{k}:=S_{k}-S_{k}=\left\{y-x \mid x, y \in S_{k}\right\}$. Then for a fixed value of the outer integration variable $\tilde{z} \in A_{k}$ we need the inner integration variable $\tilde{u}$ such that $\tilde{u} \in S_{k}$ and $\tilde{v}=\tilde{u}+\tilde{z} \in S_{k}$. In other words, for a fixed $\tilde{z} \in A_{k}$, the variable $\tilde{u}$ belongs to a $\tilde{z}$-dependent domain $E_{k}(\tilde{z}):=S_{k} \cap\left(S_{k}-\tilde{z}\right)$. This construction gives a new representation for $I$ which is equivalent to (3.13):

$$
I=\int_{\check{u} \in S_{d-k}} \int_{\check{v} \in S_{d-k}} \int_{\tilde{z} \in A_{k}} \int_{\tilde{u} \in E_{k}(\tilde{z})} H(\check{u}, \check{v}, \tilde{z}+\tilde{u}, \tilde{u}) d \tilde{u} d \tilde{z} d \check{v} d \check{u} .
$$

Step 4: Reflections. Due to [3, Lemma 3.6] we have with $M=\{1, \ldots, k\}$ the following decomposition: $A_{k}=\bigcup_{N \subset M} A_{N, M \backslash N}$, where

$$
\begin{aligned}
A_{N^{-}, N^{+}} & :=\left\{z \in A_{k} \mid z_{N^{-}} \leq 0, z_{N^{+}} \geq 0\right\} \\
& =\left\{z \mid z_{N^{-}} \in-S_{\# N^{-}}, z_{N^{+}} \in S_{\# N^{+}}\right\} .
\end{aligned}
$$

Every subdomain $A_{N^{-}, N^{+}}$admits further decomposition into a disjoint union of two pyramids $A_{N, M \backslash N}=A_{N, M \backslash N}^{+} \cup A_{N, M \backslash N}^{-}$, where

$$
\begin{aligned}
A_{N^{-}, N^{+}}^{+} & :=\left\{z \in A_{N^{-}, N^{+}} \mid \sum_{j} z_{j} \geq 0\right\} \\
& =\left\{s z \mid z_{N^{-}} \in-S_{\# N^{-}} ; z_{N^{+}} \in B_{\# N^{+}} ; s \in[0,1]\right\}, \\
A_{N^{-}, N^{+}}^{-} & :=\left\{z \in A_{N^{-}, N^{+}} \mid \sum_{j} z_{j} \leq 0\right\} \\
& =\left\{s z \mid z_{N^{-}} \in-B_{\# N^{-}} ; z_{N^{+}} \in S_{\# N^{+}} ; s \in[0,1]\right\} .
\end{aligned}
$$

Define

$$
E_{N^{-}, N^{+}}^{ \pm}(\tilde{z}):=-\tilde{z}^{\left(N^{-}\right)}+\left(1 \mp \sum_{j}\left(\tilde{z}_{N^{ \pm}}\right)_{j}\right) S_{d} .
$$

Note that $E_{N^{-}, N^{+}}^{+}(\tilde{z})$ takes the following explicit form:

$$
E_{N^{-}, N^{+}}^{+}(\tilde{z})=-\tilde{z}^{\left(N^{-}\right)}+\sigma_{\tilde{z}_{N^{+}}} S_{d}
$$

According to [3, Lemma 3.6] we have

$$
\tilde{z} \in A_{N^{-}, N^{+}}^{ \pm} \quad \text { yielding } \quad E_{k}(\tilde{z})=E_{N^{-}, N^{+}}^{ \pm}(\tilde{z}) .
$$

Copyright $@$ by SIAM. Unauthorized reproduction of this article is prohibited. 
Collecting the terms, we obtain a new representation for the integral $I$ in (3.16):

$$
I=\sum_{N \subset M} \sum_{\sigma \in\{-,+\}} \int_{\tilde{u} \in S_{d-k}} \int_{\check{v} \in S_{d-k}} \int_{\tilde{z} \in A_{N, M \backslash N}^{\sigma}} \int_{\tilde{u} \in E_{N, M \backslash N}^{\sigma}(\tilde{z})} H(\check{u}, \check{z}+\tilde{u}, \tilde{u}) d \tilde{u} d \tilde{z} d \check{v} d \check{u} .
$$

The double sum runs over $2^{k+1}-2$ many terms, because the subdomains $A_{\{\}, M}^{-}$and $A_{M,\{\}}^{+}$are trivial. Definitions (3.18), (3.19), and (3.20) directly yield

$$
A_{N^{-}, N^{+}}^{-}=-A_{N^{+}, N^{-}}^{+} \quad \text { and } \quad E_{N^{-}, N^{+}}^{-}(-\tilde{z})=\tilde{z}+E_{N^{+}, N^{-}}^{+}(\tilde{z}) .
$$

Interchanging the integration and summation over $\sigma$, we obtain from (3.23)

$$
I=\sum_{N \subsetneq M} \int_{\check{u} \in S_{d-k}} \int_{\check{v} \in S_{d-k}} \int_{\tilde{z} \in A_{N, M \backslash N}^{+}} \int_{\tilde{u} \in E_{N, M \backslash N}^{+}(\tilde{z})} f(\check{u}, \check{v}, \tilde{z}, \tilde{u}) d \tilde{u} d \tilde{z} d \check{v} d \check{u}
$$

with

$$
f(\check{u}, \check{v}, \tilde{z}, \tilde{u}):=H(\check{u}, \check{v}, \tilde{z}+\tilde{u}, \tilde{u})+H(\check{u}, \check{v}, \tilde{u}, \tilde{z}+\tilde{u}) .
$$

Note that $f$ is singular at $(\check{u}, \check{v}, \tilde{z})=0$ and is regular with respect to $\tilde{u}$.

Step 5: Permutations. Now we group the subsets $N$ with $\# N=j$ together for each $j=0, \ldots, k-1$. For every proper subset $N \subsetneq M$ of cardinality $j$ and any vector $v \in \mathbb{R}^{k}$ we define a permutation operator $P_{N}$ satisfying

$$
w=P_{N} v \quad \Leftrightarrow \quad w_{N}=\left(v_{1}, \ldots, v_{j}\right) \quad \text { and } \quad w_{M \backslash N}=\left(v_{j+1}, \ldots, v_{k}\right) .
$$

Then for $N_{j}:=\{1, \ldots, j\}, R_{j}:=\{j+1, \ldots, k\}$ there holds for any $N \subsetneq M$

$$
\left\{(\tilde{z}, \tilde{u}) \mid \tilde{z} \in A_{N, M \backslash N}^{+}, \tilde{u} \in E_{N, M \backslash N}^{+}(\tilde{z})\right\}=\left\{\left(P_{N} \tilde{z}, P_{N} \tilde{u}\right) \mid \tilde{z} \in A_{N_{j}, R_{j}}^{+}, \tilde{u} \in E_{N_{j}, R_{j}}^{+}(\tilde{z})\right\} .
$$

In other words, all domains $A_{N, M \backslash N}^{+}$and $E_{N, M \backslash N}^{+}(\tilde{z})$ with the same cardinality of the index set $N$ can be treated as images of the reference domains $A_{N_{j}, R_{j}}^{+}$and $E_{N_{j}, R_{j}}^{+}(\tilde{z})$ under a suitable coordinate permutation. As there exist only $k$ different pairs of reference domains, this allows us to rewrite (3.24) in the following equivalent form, reducing the number of distinct domains of integration. For $k=0, \ldots, d$ the integral $I$ takes the form

$$
\begin{aligned}
I & =\sum_{j=0}^{\max (k-1,0)} I_{j}, \quad \text { where } \\
I_{j} & :=\int_{\check{u} \in S_{d-k}} \int_{\check{v} \in S_{d-k}} \int_{\tilde{z} \in A_{N_{j}, R_{j}}^{+}} \int_{\tilde{u} \in E_{N_{j}, R_{j}}^{+}(\tilde{z})} g_{j}(\check{u}, \check{v}, \tilde{z}, \tilde{u}) d \tilde{u} d \tilde{z} d \check{v} d \check{u}
\end{aligned}
$$

and

$$
g_{j}(\check{u}, \check{v}, \tilde{z}, \tilde{u}):=\sum_{\substack{N \subset M, \# N=j}} f\left(\check{u}, \check{v}, P_{N} \tilde{z}, P_{N} \tilde{u}\right) .
$$

We remark that (3.27) and (3.28) formally hold also if $k=0$. In this case we necessarily have $j=0$ and

$$
I=I_{0}:=\int_{\check{u} \in S_{d}} \int_{\check{v} \in S_{d}} g_{0}(\check{u}, \check{v}) d \check{v} d \check{u} \quad \text { with } \quad g_{0}(\check{u}, \check{v}):=f(\check{u}, \check{v})
$$

Copyright (c) by SIAM. Unauthorized reproduction of this article is prohibited. 
All functions $g_{j}$ in (3.28), (3.29) have singular support $(\check{u}, \check{v}, \tilde{z})=0$ because $\tilde{z}=0$ iff $P_{N} \tilde{z}=0$.

Step 6: Transform $\tilde{\boldsymbol{u}} \in \boldsymbol{E}_{N_{j}, \boldsymbol{R}_{j}}^{+}(\tilde{z})$ to $\bar{u} \in[0,1]^{d}$. Using representation (3.21) of $E_{N_{j}, R_{j}}^{+}(\tilde{z})$ and parametrization (2.12), we introduce

$$
\begin{aligned}
& h_{j}(\check{u}, \check{v}, \tilde{z}):=\int_{\tilde{u} \in E_{N_{j}, R_{j}}^{+}(\tilde{z})} g_{j}(\check{u}, \check{v}, \tilde{z}, \tilde{u}) d \tilde{u}=\int_{\tilde{u} \in S_{k}} g_{j}\left(\check{u}, \check{v}, \tilde{z},-\tilde{z}^{\left(N_{j}\right)}+\sigma_{\tilde{z}_{R_{j}}} \tilde{u}\right) \sigma_{\tilde{z}_{R_{j}}}^{k} d \tilde{u} \\
& (3.31) \quad=\int_{\bar{u} \in[0,1]^{k}} g_{j}\left(\check{u}, \check{v}, \tilde{z},-\tilde{z}^{\left(N_{j}\right)}+\sigma_{\tilde{z}_{R_{j}}} \chi(\bar{u})\right) \sigma_{\tilde{z}_{R_{j}}}^{k} \bar{u}_{1}^{k-1} \ldots \bar{u}_{k-1} d \tilde{u}
\end{aligned}
$$

and thus

$$
I_{j}=\int_{\check{u} \in S_{d-k}} \int_{\check{v} \in S_{d-k}} \int_{\tilde{z} \in A_{N_{j}, R_{j}}^{+}} h_{j}(\check{u}, \check{v}, \tilde{z}) d \tilde{z} d \check{v} d \check{u} .
$$

Note that in this equation some of the sets $S_{d-k}, A_{N_{j}, R_{j}}^{+}$may be zero-dimensional. In this case the corresponding integration in (3.32) should be omitted. For instance, $A_{N_{j}, R_{j}}^{+}$and $E_{N_{j}, R_{j}}^{+}(\tilde{z})$ are zero-dimensional for $k=0$. In this case $j=0$ and there formally hold

$$
I_{0}=\int_{\check{u} \in S_{d}} \int_{\check{v} \in S_{d}} h_{0}(\check{u}, \check{v}) d \check{v} d \check{u}, \quad h_{0}(\check{u}, \check{v})=h_{0}(\check{u}, \check{v}, \tilde{z})=g_{0}(\check{u}, \check{v}) .
$$

The simplex $S_{d-k}$ is zero-dimensional for $k=d$, and we have

$$
I_{j}=\int_{\tilde{z} \in A_{N_{j}, R_{j}}^{+}} h_{j}(\tilde{z}) d \tilde{z}, \quad h_{j}(\tilde{z})=h_{j}(\check{u}, \check{v}, \tilde{z}) .
$$

All integrands $h_{j}$ are singular at the origin $(\check{u}, \check{v}, \tilde{z})=0$.

Step 7: Transform $(\check{u}, \check{v}, \tilde{z}) \in S_{d-k} \times S_{d-k} \times A_{N_{j}, R_{j}}^{+}$to $\left(s_{1}, a, s_{2}, b, p, s_{3}, q\right)$ $\in[\mathbf{0}, \mathbf{1}]^{\mathbf{2}-\boldsymbol{k}}$. Recall parametrizations (2.8), (2.9), and (3.18). Their combination allows us to use the following equivalent parametrizations for $\check{u}, \breve{v} \in S_{d-k}$ and $\tilde{z} \in A_{N_{j}, R_{j}}^{+}$:

$$
\begin{aligned}
\check{u} \in S_{d-k} & =\left\{\left(\sigma_{a} s_{1}, s_{1} a\right) \mid s_{1} \in[0,1], a \in S_{d-k-1}\right\}, \\
\check{v} \in S_{d-k} & =\left\{\left(\sigma_{b} s_{2}, s_{2} b\right) \mid s_{2} \in[0,1], b \in S_{d-k-1}\right\}, \\
\tilde{z} \in A_{N_{j}, R_{j}}^{+} & =\left\{\left(-s_{3} p, \sigma_{q} s_{3}, s_{3} q\right) \mid p \in S_{j}, s_{3} \in[0,1], q \in S_{k-j-1}\right\} .
\end{aligned}
$$

Depending on particular values of $j, k, d$ certain subdomains in (3.33)-(3.35) may be zero-dimensional. This gives the following cases.

Case a: $\mathbf{1} \leq \boldsymbol{k} \leq \boldsymbol{d}-\mathbf{1}$. We get for $s=\left(s_{1}, s_{2}, s_{3}\right)$

$$
I_{j}=\int_{s \in[0,1]^{3}} T_{j}(s)\left(s_{1} s_{2}\right)^{d-k-1} s_{3}^{k-1} d s_{3} d s_{2} d s_{1},
$$

where for $h_{j} \equiv h_{j}\left(\left(\sigma_{a} s_{1}, s_{1} a\right),\left(\sigma_{b} s_{2}, s_{2} b\right),\left(-s_{3} p, \sigma_{q} s_{3}, s_{3} q\right)\right)$ we set

$$
T_{j}(s):=\int_{a \in S_{d-k-1}} \int_{b \in S_{d-k-1}} \int_{p \in S_{j}} \int_{q \in S_{k-j-1}} h_{j} d q d p d b d a .
$$

Depending on $j, k, d$ we have the following structure: 
1. If $2 \leq k \leq d-2,1 \leq j \leq k-2$, then none of the subdomains in (3.37) is zero-dimensional.

2. If $0=j \leq k-2$, then $S_{j}$ is zero-dimensional and $\left(-s_{3} p, \sigma_{q} s_{3}, s_{3} q\right)=$ $\left(\sigma_{q} s_{3}, s_{3} q\right)$.

3. If $1 \leq j=k-1$, then $S_{k-j-1}$ is zero-dimensional and $\left(-s_{3} p, \sigma_{q} s_{3}, s_{3} q\right)=$ $\left(-s_{3} p\right)$.

4. If $k=1$, then both subdomains $S_{j}$ and $S_{k-j-1}$ are zero-dimensional and $\left(-s_{3} p, \sigma_{q} s_{3}, s_{3} q\right)=\left(s_{3}\right)$. Note that $s_{3}^{k-1}=1$ in (3.36) in this case.

5 . If $k=d-1$, then $S_{d-k-1}$ is zero-dimensional and $\left(\left(\sigma_{a} s_{1}, s_{1} a\right),\left(\sigma_{b} s_{2}, s_{2} b\right)\right)=$ $\left(s_{1}, s_{2}\right)$. Note that $\left(s_{1} s_{2}\right)^{d-k-1}=1$ in $(3.36)$ in this case.

We use the parametrization of a simplex by a cube from (2.11) and (2.12), $a=$ $\chi(\bar{a})$, and similarly for $b, p, q$. Then $T_{j}(s)$ from (3.36) reads

$T_{j}(s)=\int_{\bar{a} \in[0,1]^{d-k-1}} \int_{\bar{b} \in[0,1]^{d-k-1}} \int_{\bar{p} \in[0,1]^{j}} \int_{\bar{q} \in[0,1]^{k-j-1}} h_{j} J_{\chi}(\bar{a}) J_{\chi}(\bar{b}) J_{\chi}(\bar{p}) J_{\chi}(\bar{q}) d \bar{q} d \bar{p} d \bar{b} d \bar{a}$

with the function

$$
h_{j} \equiv h_{j}\left(\left(\sigma_{\chi(\bar{a})} s_{1}, s_{1} \chi(\bar{a})\right),\left(\sigma_{\chi}(\bar{b}) s_{2}, s_{2} \chi(\bar{b})\right),\left(-s_{3} \chi(\bar{p}), \sigma_{\chi(\bar{q})} s_{3}, s_{3} \chi(\bar{q})\right)\right)
$$

and monomial weights $J_{\chi}$ defined in (2.15).

Case b: $\boldsymbol{k}=\mathbf{0}$. In this case $A_{N_{j}, R_{j}}^{+}$is zero-dimensional and we get for $s=\left(s_{1}, s_{2}\right)$

$$
\begin{aligned}
I_{0}= & \int_{s \in[0,1]^{2}} T_{0}(s)\left(s_{1} s_{2}\right)^{d-1} d s_{2} d s_{1}, \quad \text { with } \\
T_{0}(s):= & \int_{\bar{a} \in[0,1]^{d-1}} \int_{\bar{b} \in[0,1]^{d-1}} h_{0}\left(\left(\sigma_{\chi(\bar{a})} s_{1}, s_{1} \chi(\bar{a})\right),\left(\sigma_{\chi(\bar{b})} s_{2}, s_{2} \chi(\bar{b})\right)\right) \\
& \cdot J_{\chi}(\bar{a}) J_{\chi}(\bar{b}) d \bar{b} d \bar{a} .
\end{aligned}
$$

Case c: $\boldsymbol{k}=\boldsymbol{d}$. Then $S_{d-k} \times S_{d-k}$ is zero-dimensional and we get for $s=s_{3}$

$$
I_{j}=\int_{s_{3} \in[0,1]} T_{j}\left(s_{3}\right) s_{3}^{d-1} d s_{3},
$$

where

$$
T_{j}\left(s_{3}\right)=\int_{\bar{p} \in[0,1]^{j}} \int_{\bar{q} \in[0,1]^{k-j-1}} h_{j}\left(-s_{3} \chi(\bar{p}), \sigma_{\chi(\bar{q})} s_{3}, s_{3} \chi(\bar{q})\right) J_{\chi}(\bar{p}) J_{\chi}(\bar{q}) d \bar{q} d \bar{p} .
$$

Depending on values of $j, k, d$, cases a.1-a.4 are valid here. We remark that all integrands $T_{j}$ in cases $\mathbf{a}, \mathbf{b}$, and $\mathbf{c}$ are singular iff $s=0$.

Step 8: Integration over $s$ in a cube.

Case a: $\mathbf{1} \leq \boldsymbol{k} \leq \boldsymbol{d}-\mathbf{1}$. Here $I_{j}$ in (3.36) reads

$$
\begin{aligned}
I_{j}= & \int_{r \in[0,1]^{3}}\left(T_{j}\left(r_{1}, r_{1} r_{2}, r_{1} r_{3}\right)+T_{j}\left(r_{1} r_{2}, r_{1}, r_{1} r_{3}\right)\right) r_{1}^{2 d-k-1} r_{2}^{d-k-1} r_{3}^{k-1} \\
& +\left(T_{j}\left(r_{1} r_{2}, r_{1} r_{3}, r_{1}\right)\right) r_{1}^{2 d-k-1}\left(r_{2} r_{3}\right)^{d-k-1} d r_{3} d r_{2} d r_{1} .
\end{aligned}
$$

Case b: $\boldsymbol{k}=\mathbf{0}$. Then $I_{j}$ in (3.36) takes the form

$$
I_{j}=\int_{r \in[0,1]^{2}}\left(T_{j}\left(r_{1}, r_{1} r_{2}\right)+T_{j}\left(r_{1} r_{2}, r_{1}\right)\right) r_{1}^{2 d-1} r_{2}^{d-1} d r_{2} d r_{1}
$$

Copyright $@$ by SIAM. Unauthorized reproduction of this article is prohibited. 
Case c: $\boldsymbol{k}=\boldsymbol{d}$. In this case no transform is needed, and we get

$$
I_{j}=\int_{r_{1} \in[0,1]} T_{j}\left(r_{1}\right) r_{1}^{d-1} d r_{1} .
$$

Note that the integrands in all three cases are singular only in $r_{1}=0$ and regular in the remaining variables.

Remark 2. In summary, we have rewritten the integral (1.3) as

$$
I=\sum_{j=0}^{\min (k-1,0)} I_{j}, \quad I_{j}=\int_{t \in[0,1]^{2 d}} \sum_{\kappa=1}^{K_{j}}\left(F \circ \Phi_{\kappa j}\right)(t) J_{\Phi_{\kappa j}}(t) d t .
$$

Enumeration with a single summation index $(\kappa, j) \rightarrow \varkappa$ brings (3.46) into the desired form (3.1). Hence, the quadrature strategy described in the beginning of the present section can be applied. Here, $t$ stands for the collection of all integration variables in the unit cube $[0,1]^{2 d}$ after the transformations in Steps $1-8$, i.e., $2 d$ variables $r, \bar{a}, \bar{b}, \bar{p}, \bar{q}, \bar{u}$. We identify $t_{1}=r_{1}$ as the singular variable.

Remark 3. We observe that the Jacobians can be factored, i.e.,

$$
J_{\Phi_{\kappa j}}(t)=t_{1}^{2 d-k-1} J_{\Phi_{\kappa j}}^{\mathrm{mon}}\left(t_{2}, \ldots, t_{2 d}\right) J_{\Phi_{\kappa j}}^{\mathrm{rem}}(t),
$$

where $J_{\Phi_{\kappa j}}^{\text {mon }}$ is a product of monomials and $J_{\Phi_{\kappa j}}^{\text {rem }}$ is a polynomial remainder. Precisely,

$$
J_{\Phi_{\kappa j}}^{\text {mon }}=\left\{\begin{array}{cl}
J_{\chi}(\bar{u}) J_{\chi}(\bar{v}), & k=-1, \\
r_{2}^{d-1} J_{\chi}(\bar{a}) J_{\chi}(\bar{b}), & k=0, \\
\left\{\begin{array}{c}
r_{2}^{d-k-1} r_{3}^{k-1} J_{\chi}(\bar{a}) J_{\chi}(\bar{b}) J_{\chi}(\bar{p}) J_{\chi}(\bar{q}) J_{\chi}(\bar{u}) \\
\text { and } \\
\left(r_{2} r_{3}\right)^{d-k-1} J_{\chi}(\bar{a}) J_{\chi}(\bar{b}) J_{\chi}(\bar{p}) J_{\chi}(\bar{q}) J_{\chi}(\bar{u}) \\
J_{\chi}(\bar{p}) J_{\chi}(\bar{q}) J_{\chi}(\bar{u}),
\end{array}\right. & 1 \leq k \leq d, \\
& k=d,
\end{array}\right.
$$

where, according to (2.13),

$$
J_{\chi}:\left\{\begin{aligned}
{[0,1]^{m} } & \rightarrow \mathbb{R} \\
t & \mapsto t_{1}^{m-1} \ldots t_{m-1} .
\end{aligned}\right.
$$

Note the following:

- The singular coordinate $t_{1}$ is not contained in $J_{\Phi_{\kappa j}}^{\mathrm{mon}}$.

- In the case $k>0$, the dimension of $\bar{a}, \bar{b}, \bar{p}, \bar{q}$, and hence the exact form of the Jacobians in (3.48) depends on the parameter $j$.

- The splitting (3.47) follows solely from the explicit form of the coordinate transforms in Steps 1-8 and not from the particular assumptions on the integrand. Hence it is valid, e.g., for the general class of admissible integrands satisfying (1.4).

Having the splitting (3.47), the monomial part $J_{\Phi_{\kappa j}}$ can be incorporated into the quadrature weights in terms of the tensor product Gauss-Jacobi quadrature. Suppose $\left\{\left(t_{\mu \kappa j}^{G J}, w_{\mu \kappa j}^{G J}\right)\right\}_{\mu=1}^{n}$ is the corresponding quadrature rule, i.e.,

$$
\int_{t \in[0,1]^{2 d}} F_{1}(t) J_{\Phi_{\kappa j}}^{\mathrm{mon}}\left(t_{2}, \ldots, t_{2 d}\right) d t \approx \sum_{\mu} F_{1}\left(t_{\mu \kappa j}^{G J}\right) w_{\mu \kappa j}^{G J}
$$

Copyright $@$ by SIAM. Unauthorized reproduction of this article is prohibited. 
for some sufficiently smooth $F_{1}$. Then

$$
I \approx Q_{N}^{G J}:=\sum_{\mu=1}^{n} \sum_{j=0}^{\min (k-1,0)} \sum_{\kappa=1}^{K_{j}} F\left(x_{\mu \kappa j}, y_{\mu \kappa j}, z_{\mu \kappa j}\right) w_{\mu \kappa j},
$$

where $N=n\left(K_{0}+\cdots+K_{\min (k-1,0)}\right)$ and

$$
\left(x_{\mu \kappa j}, y_{\mu \kappa j}, z_{\mu \kappa j}\right)=\Phi_{\kappa j}\left(t_{\mu \kappa j}^{G J}\right), \quad w_{\mu \kappa j}=\left.t_{1}^{2 d-k-1} J_{\Phi_{\kappa j}}^{\mathrm{rem}}(t) w_{\mu \kappa j}^{G J}\right|_{t=t_{\mu \kappa j}^{G J}} .
$$

Note that $J_{\Phi_{\kappa j}}^{\text {mon }}=t^{\beta_{(\kappa j)}}$ for a suitable multi-index $\beta_{(\kappa j)}=\left(0, \beta_{2}, \ldots, \beta_{2 d}\right)$ with integer entries. In Theorems 5.3 and 5.4 we prove exponential convergence for this type of quadrature rule.

Remark 4. In the case of volume or surface layer potentials for second order elliptic operators there are two possible cases:

1. The singularity of $F(x, y, z)$ is of the type $\|z\|^{\alpha}$ with integer $\alpha$. More precisely, the integrand satisfies [10, section 5.1.3]

$$
F(x, y, z)=\|z\|^{\alpha} \tilde{F}(x, y, z), \quad \tilde{F}(x, y, z)=\hat{F}(x, y,\|z\|, z /\|z\|),
$$

$\hat{F}(x, y, r, \theta)$ analytic in $x \in S^{(1)}, y \in S^{(2)}, r \in[0, R], \theta \in\left\{z \in \mathbb{R}^{d} \mid\|z\|=1\right\}$.

After applying the transformations in Steps 1-8, the integrands will become analytic in all variables; see [3, Remark 4.3]. In this case the variable $t_{1}$ can be treated the same as the remaining variables. In this case the full tensor product Gauss-Legendre quadrature rule achieves exponential convergence (1.6) with $\gamma=1 /(2 d \delta)$ for all $k=-1,0, \ldots, d$ instead of $\gamma=1 /(2 d \delta+1)$ if a composite Gauss-Legendre quadrature rule [11] is used in $t_{1}$ [3, Theorem 4.5].

2. For elliptic PDEs in two dimensions the fundamental solution has a logarithmic singularity. Therefore, single layer potentials and volume potentials have a logarithmic singularity, and $F(x, y, z)$ has a singularity of type $\|z\|^{\alpha_{0}} \log \|z\|$ with integer $\alpha_{0}$. In this case (1.4) is satisfied with $\alpha=\alpha_{0}-\varepsilon$ for any $\varepsilon>0$.

We prove in Theorem 5.4 below that an exponential convergence with $\gamma=1 /(2 d \delta)$ can be also achieved for integrands of the type (3.49) and noninteger $\alpha>k-2 d$ with the aid of a suitable Gauss-Jacobi quadrature rule applied in the $t_{1}$-direction.

4. Explicit structure of the transformed singularity $F$ satisfying (1.7). The main result of this section is the proof of the following theorem.

TheOREM 4.1. Suppose $k=\operatorname{dim}\left(S^{(1)} \cap S^{(2)}\right) \geq 0$ and the integrand $F$ in (1.3) has the structure

$$
F(x, y, z)=\|z\|^{\alpha} \tilde{F}(x, y, z),
$$

where $\alpha>k-2 d$ and $\tilde{F} \in G^{\delta}\left(S^{(1)} \times S^{(2)} \times\left(S^{(2)}-S^{(1)}\right)\right)$ for some $\delta \geq 1$. Then after the coordinate transforms from section 3 , the integral I in (1.3) takes the form

$$
I=\int_{[0,1]^{2 d}} t_{1}^{\alpha+2 d-k-1} \mathcal{R}(t) d t
$$

with some $\mathcal{R} \in G^{\delta}\left([0,1]^{2 d}\right)$.

Proof. It suffices to prove that under the coordinate transforms in section 3 the function

$$
\frac{\|y(t)-x(t)\|^{\alpha}}{t_{1}^{\alpha}}
$$

Copyright $@$ by SIAM. Unauthorized reproduction of this article is prohibited. 
is analytic in $t \in[0,1]^{2 d}$. Then the assertion follows by Propositions A.1 and A.2 and the fact that the coordinate transformations in section 3 are polynomial and hence analytic, i.e., belong to $G^{1}\left([0,1]^{2 d}\right)$. The regularizing effect of the coordinate transforms in section 3 is due to the Jacobian determinant $t_{1}^{2 d-k-1}$, appearing in (3.43)-(3.45). The transformations are rather complex, and we split

$$
\frac{\|y-x\|^{\alpha}}{t_{1}^{\alpha}}=\frac{\|y-x\|^{\alpha}}{\|(\check{u}, \check{v}, \hat{v}-\hat{u})\|^{\alpha}} \frac{\|(\check{u}, \check{v}, \hat{v}-\hat{u})\|^{\alpha}}{\|(\check{u}, \check{v}, \tilde{z})\|^{\alpha}} \frac{\|(\check{u}, \check{v}, \tilde{z})\|^{\alpha}}{\|s\|^{\alpha}} \frac{\|s\|^{\alpha}}{t_{1}^{\alpha}}=: \mathcal{A}_{1} \mathcal{A}_{2} \mathcal{A}_{3} \mathcal{A}_{4}
$$

for the sake of convenience. We show analyticity of all four fractions in the right-hand side separately. We follow here the strategy in [10, Chapter 5], $[12,11]$ restricted to the special case $d=2$. We represent each $\mathcal{A}_{i}$ in the form $\mathcal{A}_{i}=\|\omega\|^{\alpha}$ for some suitable analytic (in fact, polynomial) $\omega$ and show that $\omega$ has no real roots in the integration domain. This implies analyticity of $\mathcal{A}_{i}$.

(i) We introduce polar coordinates $(r, \theta)$ satisfying

$$
\zeta:=(\check{u}, \check{v}, \hat{v}-\hat{u})=r \theta, \quad r=\|\zeta\|, \quad \theta=\frac{\zeta}{\|\zeta\|}=\left(\theta^{(1)}, \theta^{(2)}, \theta^{(3)}\right) .
$$

The aim is to show that $\mathcal{A}_{1}=\mathcal{A}_{1}(\theta)$ is analytic in the polar coordinates. Here the subvector $\theta^{(3)}$ is empty if $k=0$ and $\theta^{(1)}, \theta^{(2)}$ are empty if $k=d$. Recalling transformation (3.5), we find

$$
y-x=r \omega(\theta), \quad \omega(\theta)=\left\{\begin{array}{rc}
\theta^{(2)} B^{(2)}-\theta^{(1)} B^{(1)}, & k=0 \\
\theta^{(3)} B+\theta^{(2)} B^{(2)}-\theta^{(1)} B^{(1)}, & 0<k<d, \\
\theta^{(3)} B, & k=d .
\end{array}\right.
$$

Note that $\omega(\theta)$ is a linear map and hence is analytic. According to [3, (3.9)] there exists $c_{1}>0$ such that $\|y-x\| \geq c_{1}\|\zeta\|=c_{1} r$, yielding

$$
\|\omega(\theta)\|=\frac{\|y-x\|}{r} \geq c_{1}>0 .
$$

Then, $\omega(\theta)$ is never zero for $\theta$ corresponding to $(\hat{u}, \check{u}),(\hat{v}, \check{v}) \in S_{d}$, and hence $\mathcal{A}_{1}=$ $\frac{\|y-x\|^{\alpha}}{r^{\alpha}}=\|\omega(\theta)\|^{\alpha}$ is analytic. This covers the coordinate transformation in Step 1.

(ii) If $k=0$, we have $\mathcal{A}_{2}=1$. Suppose $0<k \leq d$. Let us redefine the polar coordinates

$$
\zeta:=(\check{u}, \check{v}, \tilde{z})=r \theta, \quad r=\|\zeta\|, \quad \theta=\frac{\zeta}{\|\zeta\|}=\left(\theta^{(1)}, \theta^{(2)}, \theta^{(3)}\right)
$$

and prove that $\mathcal{A}_{2}$ is analytic as a function of $r, \theta, \tilde{u}$. Recalling transformations in Step 2 of section 3, in particular relation (3.15), we find $(\check{u}, \breve{v}, \hat{v}-\hat{u})=r \omega(r, \theta, \tilde{u})$, where

$$
\omega(r, \theta, \tilde{u})=\left\{\begin{array}{cc}
\left(\theta^{(1)}, \theta^{(2)}, \theta^{(3)} \sigma_{\left(r \theta^{(2)}\right)}+\tilde{u} \sum_{j=1}^{d-k}\left(\theta^{(1)}-\theta^{(2)}\right)\right), & 0<k<d, \\
\theta^{(3)}, & k=d .
\end{array}\right.
$$

Lemma 4.12 from [3] provides the estimate

$$
\|\omega(r, \theta, \tilde{u})\|=\frac{\|(\check{u}, \check{v}, \hat{v}-\hat{u})\|}{r} \geq c_{2}>0
$$

Copyright $\odot$ by SIAM. Unauthorized reproduction of this article is prohibited. 
uniformly in the domain of integration. Therefore, the polynomial $\omega(r, \theta, \tilde{u})$ is nonzero if $(\tilde{u}, \check{u}),(\tilde{v}, \check{v}) \in S_{k} \times S_{d-k}$; thus $\mathcal{A}_{2}=\frac{\|(\check{u}, \breve{v}, \hat{v}-\hat{u})\|^{\alpha}}{r^{\alpha}}=\|\omega(r, \theta, \tilde{u})\|^{\alpha}$ is analytic. This result covers the transformations in Step 2. In Steps 3-5 only domain partition happens, and the transformation in Step 6 does not influence the singularity. This gives the desired result for transformations in Steps 2-6.

(iii) Let us consider the function $\mathcal{A}_{3}$. For

$$
s=\left\{\begin{array}{cc}
\left(s_{1}, s_{2}\right), & k=0 \\
\left(s_{1}, s_{2}, s_{3}\right), & 0<k<d \\
s_{3}, & k=d,
\end{array}\right.
$$

we introduce polar coordinates via the relation

$$
s=r \theta, \quad r=\|s\|, \quad \theta=\frac{s}{\|s\|}=\left\{\begin{array}{cc}
\left(\theta_{1}, \theta_{2}\right), & k=0 \\
\left(\theta_{1}, \theta_{2}, \theta_{3}\right), & 0<k<d \\
\theta_{3}, & k=d
\end{array}\right\}
$$

and show that $\mathcal{A}_{3}=\mathcal{A}_{3}(\theta)$ is an analytic function is polar coordinates. Combining all the transformations in Step 7, we obtain

$$
(\check{u}, \check{v}, \tilde{z})=r \omega(\theta, a, b, p, q), \quad \omega(\theta, a, b, p, q)=\left(\sigma_{a} \theta_{1}, \theta_{1} a, \sigma_{b} \theta_{2}, \theta_{2} b,-\theta_{3} p, \sigma_{q} \theta_{3}, \theta_{3} q\right),
$$

where some components of $\omega$ may vanish depending on the values of $j, k, d$; cf. Step 7 . For the $\|\cdot\|_{1}$-norm we have

$$
\|\omega\|_{1} \geq\|\theta\|_{1} \min \left\{\left\|\left(\sigma_{a}, a\right)\right\|_{1},\left\|\left(\sigma_{b}, b\right)\right\|_{1},\left\|\left(\sigma_{q}, q\right)\right\|_{1}\right\} \geq\|\theta\|_{1}
$$

because $\left\|\left(\sigma_{a}, a\right)\right\|_{1}=\left|1-\sum a_{j}\right|+\sum\left|a_{j}\right| \geq 1$. Since in a finite dimensional space all norms are equivalent, we have $\|\omega\| \geq c_{3}(d)\|\theta\|=c_{3}(d)>0$ uniformly in the domain of integration, and hence $\omega$ is nonzero. Hence $\mathcal{A}_{3}=\frac{\|(\breve{u}, \tilde{v}, \tilde{z})\|^{\alpha}}{r^{\alpha}}=\|\omega\|^{\alpha}$ is analytic. This covers the coordinate transformations in Step 7.

(iv) Finally, we prove that $\mathcal{A}_{4}$ is analytic. In Step 8 the variables $s$ are transformed to variables $r$ according to (3.43)-(3.45), and $r_{1}$ is identified with $t_{1}$. In all three cases we have $s=t_{1} \omega(r)$ with

$$
\omega(r)=\left\{\begin{array}{cc}
\left(1, r_{2}\right), & k=0 \\
\left(1, r_{2}, r_{3}\right), & 0<k<d \\
1, & k=d
\end{array}\right.
$$

up to a permutation. Therefore, $\|\omega(r)\| \geq 1$, yielding that $\mathcal{A}_{4}=\frac{\|s\|^{\alpha}}{t_{1}^{\alpha}}=\|\omega(r)\|^{\alpha}$ is an analytic function of $r$. The proof is complete.

5. Convergence of Gauss-Jacobi quadrature rules. In this section we give convergence results for tensor product quadratures involving one-dimensional GaussJacobi rules.

5.1. Convergence of the Gauss-Jacobi quadrature for Gevrey- $\delta$ integrands on an interval. Suppose $\alpha, \beta>-1$ are fixed parameters, and consider a continuous function $g$ on an open interval $(a, b)$ such that $\int_{a}^{b}|g(x)|(b-x)^{\alpha}(x-a)^{\beta} d x<\infty$. We approximate the integral

$$
I^{[a, b],(\alpha, \beta)}[f]:=\int_{a}^{b} g(x)(b-x)^{\alpha}(x-a)^{\beta} d x
$$


by the Gauss--Jacobi quadrature rule:

$$
Q_{n}^{[a, b],(\alpha, \beta)}[f]:=\sum_{i=1}^{n} g\left(x_{i}\right) w_{i}
$$

The Gauss-Jacobi quadrature rule is exact for polynomials of degree up to $2 n-1$; cf. [8, Chapter 7.3].

TheOREm 5.1. Let $n \in \mathbb{N}$ and $k \in\{2,3, \ldots, 2 n-1\}$. Then for $g \in C^{k}([a, b])$

$$
\begin{aligned}
& \left|I^{[a, b],(\alpha, \beta)}[g]-Q_{n}^{[a, b],(\alpha, \beta)}[g]\right| \\
& \quad \leq\left(\frac{b-a}{2}\right)^{\alpha+\beta+k+1} \frac{\Gamma(\alpha+1) \Gamma(\beta+1)}{\Gamma(\alpha+\beta+2)} \frac{2^{\alpha+\beta+3}\left\|g^{(k)}\right\|_{\infty}}{(k-1)(2 n-k)^{k-1}} .
\end{aligned}
$$

Proof. We first consider $[a, b]=[-1,1] . Q_{n}^{[-1,1],(\alpha, \beta)}$ is exact of degree $2 n-1$; hence for an arbitrary polynomial $\psi$ of degree up to $2 n-1$

$$
\begin{aligned}
\left|I^{[-1,1],(\alpha, \beta)}[g]-Q_{n}^{[-1,1],(\alpha, \beta)}[g]\right| & \leq\left|I^{[-1,1],(\alpha, \beta)}[g-\psi]\right|+\left|Q_{n}^{[-1,1],(\alpha, \beta)}[g-\psi]\right| \\
& \leq 2 I^{[-1,1],(\alpha, \beta)}[1]\|g-\psi\|_{\infty}
\end{aligned}
$$

and $I^{[-1,1],(\alpha, \beta)}[1]=2^{\alpha+\beta+1} \Gamma(\alpha+1) \Gamma(\beta+1) / \Gamma(\alpha+\beta+2)<\infty$, since $\alpha, \beta>-1$. We choose a particular $\psi:=T_{2 n-1}[g]$ as the Chebyshev approximation of degree $2 n-1$ to $g$. According to [14, Theorem 4.3] this yields the bound

$$
\|g-\psi\|_{\infty} \leq \frac{2\left\|g^{(k)}(x) / \sqrt{1-x^{2}}\right\|_{1}}{\pi(k-1)(2 n-k)^{k-1}} \leq \frac{2\left\|g^{(k)}\right\|_{\infty}}{(k-1)(2 n-k)^{k-1}} .
$$

An affine transformation to $[a, b]$ yields the assertion.

Theorem 5.2. Assume that $g \in G^{\delta}([a, b])$ with $\delta \geq 1$ and constants $A_{0}, A_{1}$ in (1.2). Let $\rho:=A_{1}(b-a) / 2$. Let

$$
r_{*}:=\left\{\begin{array}{cc}
2 \log \left[\rho^{-1}+\sqrt{1+\rho^{-2}}\right], & \delta=1, \\
\delta \rho^{-1 / \delta}, & \delta>1 .
\end{array}\right.
$$

Then for any $r<r_{*}$ there exists $C>0$ depending only on $r, \rho, \alpha, \beta$, and $\delta$ such that for all $n \in \mathbb{N}$

$$
\left|I^{[a, b],(\alpha, \beta)}[g]-Q_{n}^{[a, b],(\alpha, \beta)}[g]\right| \leq A_{0}(b-a)^{\alpha+\beta+1} C \exp \left(-r n^{1 / \delta}\right) .
$$

Proof. The proof is analogous to [3, Theorem 2.3]. In the case $\delta=1$ the only difference is the factor $\left(\frac{b-a}{2}\right)^{\alpha+\beta+1}$ appearing while scaling $[a, b]$ to $[-1,1]$ in $[3,(2.4)]$. In the case $\delta>1$ the expression $\frac{32}{15} \frac{b-a}{2}$ in $[3,(2.5)]$ changes to $\left(\frac{b-a}{2}\right)^{\alpha+\beta+1} 2^{\alpha+\beta+3}$ . $\frac{\Gamma(\alpha+1) \Gamma(\beta+1)}{\Gamma(\alpha+\beta+2)}$ according to Theorem 5.1.

5.2. Tensor product quadrature on $[0,1]^{d}$. The following theorems follow directly from Theorem 5.2, two theorems from [3], [3, Theorems 2.5 and 2.7], and the tensor product argument in [3, Proposition 2.8].

Copyright $@$ by SIAM. Unauthorized reproduction of this article is prohibited. 
TheOREM 5.3. Suppose $d \in \mathbb{N}, \delta \geq 1$, and $\alpha>-1$, and assume that $g \in$ $G_{\{1\}}^{\delta, \alpha}\left([0,1]^{d}\right)$. Let $Q_{n, m}$ be the constant or variable order composite Gauss-Legendre quadrature rule on $[0,1]$ with $m$ subintervals and $n$ nodes in the largest subinterval, $m \sim n^{1 / \delta} ;$ cf. [3, Definition 2.4]. Let $\beta=\left(0, \beta_{2}, \ldots, \beta_{d}\right)$ be a multi-index satisfying $\beta>-1$ and $Q_{n}^{\beta_{i}}=Q_{n}^{[0,1],\left(0, \beta_{i}\right)}$ be a family of Gauss-Jacobi quadratures with $n$ nodes. Then there exist $C, r, r^{\prime}>0$ independent of $d$ such that for all $n \in \mathbb{N}$

$$
\begin{aligned}
\left|\int_{[0,1]^{d}} g(t) t^{\beta} d t-Q_{n, m} \otimes Q_{n}^{\beta_{2}} \otimes \cdots \otimes Q_{n}^{\beta_{d}}[g]\right| & \leq C \exp \left(-r n^{1 / \delta}\right) \\
& \leq C \exp \left(-r^{\prime} N^{1 /(d \delta+1)}\right),
\end{aligned}
$$

where $N=\mathcal{O}\left(n^{d+\delta^{-1}}\right)$ is the total number of quadrature nodes.

Theorem 5.4. Suppose $d \in \mathbb{N}$ and $\delta \geq 1$, and assume that $g \in G^{\delta}\left([0,1]^{d}\right)$. Let $\beta=\left(\beta_{1}, \beta_{2}, \ldots, \beta_{d}\right)$ be a multi-index satisfying $\beta>-1$, and let $Q_{n}^{\beta_{i}}=Q_{n}^{[0,1],\left(0, \beta_{i}\right)}$ be a family of Gauss-Jacobi quadratures with $n$ nodes. Then there exist $C, r, r^{\prime}>0$ independent of $d$ such that for all $n \in \mathbb{N}$

$$
\left|\int_{[0,1]^{d}} g(t) t^{\beta} d t-Q_{n}^{\beta_{1}} \otimes Q_{n}^{\beta_{2}} \otimes \cdots \otimes Q_{n}^{\beta_{d}}[g]\right| \leq C \exp \left(-r n^{1 / \delta}\right) \leq C \exp \left(-r^{\prime} N^{1 /(d \delta)}\right),
$$

where $N=\mathcal{O}\left(n^{d}\right)$ is the total number of quadrature nodes.

6. Numerical examples. In this section we give convergence results for the suggested $2 d$-dimensional quadratures involving one-dimensional Gauss--Jacobi quadrature rules and compare them to the $h p$-quadratures from [3].

6.1. Comparison of composite Gauss-Legendre and Gauss-Jacobi rules for Gevrey- $\delta$ integrands with singularity in one dimension. In this section we compare performance of the composite Gauss-Legendre quadrature and the GaussJacobi quadrature for a Gevrey- $\delta$ integrand with an algebraic singularity on an interval. Let us consider a function

$$
f_{\alpha, p}(x)=(1-x)^{\alpha} g_{p}(x), \quad g_{p}(x)=\left\{\begin{array}{lr}
\exp \left(-x^{-p}\right), & 0<x \leq 1, \\
0, & -1 \leq x \leq 0 .
\end{array}\right.
$$

The function $f_{\alpha, p}$ has an integrable singularity at 1 if $\alpha>-1$, and is not analytic in any neighborhood of 0 . Precisely, $g_{p} \in G^{\delta}([-1,1])$ iff $\delta \geq 1+1 / p$; cf. [3]. According to Theorem 5.4, we expect the convergence rate of order $\mathcal{O}\left(\exp \left(-r^{\prime} N^{1 / \delta}\right)\right)$ for the GaussJacobi rule with the weight $(\alpha, 0)$. According to [3], we expect the convergence of order $\mathcal{O}\left(\exp \left(-r^{\prime} N^{1 /(2 \delta)}\right)\right)$ for the composite Gauss-Legendre rule. Convergence results for $p=2$ and $\alpha=1 / \pi-1$ given in Figure 6.1 show the sharpness of these estimates. We observe a clear advantage of the Gauss-Jacobi quadrature rule for integration of this type of singularity if the exponent $\alpha$ is known. The classical Gauss-Legendre quadrature rule converges slowly, because the integrand $f_{\alpha, p}$ has a singularity; see, e.g., $[5]$.

6.2. Performance of Gauss-Jacobi quadrature rules for $2 d$-dimensional integrands. We consider the integral (1.3) for two particular simplices $S^{(1)}:=S_{d}$ and $S^{(2)}:=\left(\left(y_{1}, \ldots, y_{k},-y_{k+1}, \ldots,-y_{d}\right) \mid y \in S_{d}\right)$, where $k=0, \ldots, d$, i.e., the intersection $S^{(1)} \cap S^{(2)}=\left\{(\hat{x}, 0, \ldots, 0) \mid \hat{x} \in S_{k}\right\}$ is of dimension $k$. For $k=-1$ we use $S^{(1)}:=S_{d}$ and $S^{(2)}:=S_{d}-(1, \ldots, 1)$. 


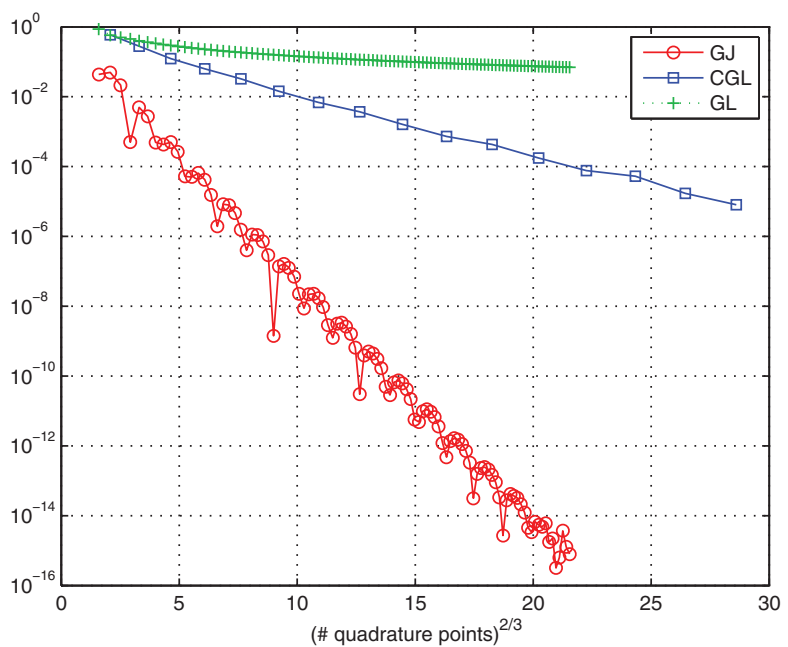

FIG. 6.1. Comparison of Gauss-Jacobi, composite Gauss-Legendre, and Gauss-Legendre quadrature rules for the univariate Gevrey- $\delta$ integrand with an algebraic singularity from (6.1). kernel

We examine the performance of various transformed quadrature rules for the

$$
F(x, y, z)=\|z\|^{\alpha}, \quad z=y-x, \quad \alpha=k-2 d+\beta
$$

with $\beta>0$; i.e., the exponent is larger by $\beta$ than the critical exponent $k-2 d$, where $F(x, y, y-x) \notin L^{1}\left(S^{(1)} \times S^{(2)}\right)$. Note that $F$ satisfies assumptions (1.4) and (1.7). In this case, quadrature rules involving a suitable Gauss-Jacobi rule in the singular direction are expected to be more efficient than those using the general purpose composite Gauss-Legendre rule.

In our numerical experiments we used $\beta=1 / \pi$. We choose singularities very close to the nonintegrable case for all $k=0, \ldots, d$ as a severe test for our algorithm. In applications for integral equations one has a fixed integrand $F(x, y, z) \sim\|z\|^{\alpha_{*}}$ with $\alpha_{*}>-d$ for all $k$, and $\beta=\beta_{k}:=\alpha_{*}-(k-2 d)>d-k$.

As motivated in the beginning of section 3, any sufficiently accurate (e.g., exponentially converging) quadrature rule on the cube $t \in[0,1]^{2 d}$ for integrands with univariate algebraic singularity in $t_{1}$ and Gevrey- $\delta$ smooth in $\left(t_{2}, \ldots, t_{2 d}\right)$ yields an accurate (exponentially converging) quadrature rule in the physical domain by means of the transform in section 3. In what follows we compare three classes of tensor product quadrature rules corresponding to the following combination in the singular/regular directions on $[0,1]^{2 d}$ :

1. composite Gauss-Legendre/Gauss-Legendre (CGL-GL),

2. Gauss-Jacobi/Gauss-Legendre (GJ-GL),

3. Gauss-Jacobi/Gauss-Jacobi (GJ-GJ).

In the tests we use the Gauss-Legendre and Gauss-Jacobi quadrature rules with $n$ nodes in each direction. The parameters of the composite Gauss-Legendre quadrature rule are chosen as follows. Let $\tilde{n}=2 n$. We use a geometric mesh with ratio $\sigma=0.1$ and $m=\tilde{n}$ subintervals, with $1,2, \ldots, \tilde{n}$ Gauss-Legendre points on the subintervals; cf. [3] for the details.

Other combinations of quadrature rules are also possible. We refer the reader to [2] for more numerical examples, combining, e.g., Smolyak-type quadrature rules in the 

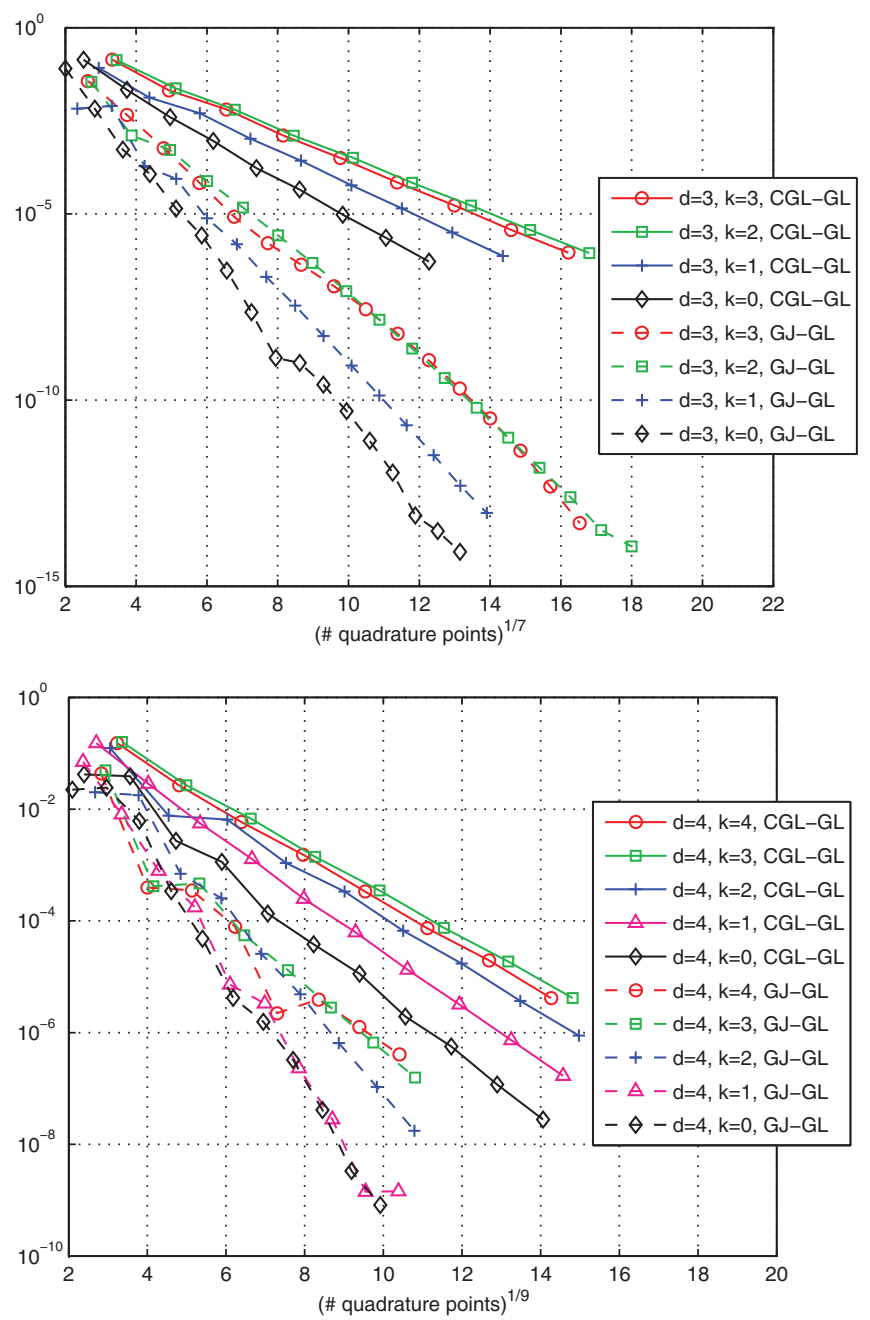

FIG. 6.2. Comparison of $C G L$ and $G J$ in the singular direction for $F=\|y-x\|^{\alpha}$. Relative error $\left|I-Q_{N}\right| /|I|$ for $d=3,4$ and $k=0, \ldots, d$.

"regular" direction with the Gauss-Jacobi or composite Gauss-Legendre quadrature rule in the "singular" direction.

In the convergence plots we show on the vertical axis the relative error $\left|I-Q_{N}\right| /|I|$ in the logarithmic scale, and on the horizontal axis $N^{1 /(2 d+1)}$, where $N$ is the total number of quadrature points (or function evaluations). This yields

$$
N=\left\{\begin{array}{cl}
K \frac{\tilde{n}(\tilde{n}+1)}{2} n^{2 d-1}, & 0 \leq k \leq d, \\
n^{2 d}, & k=-1,
\end{array} \quad K= \begin{cases}2 & \text { for } k=0, \\
3\left(2^{k+1}-2\right) & \text { for } 1 \leq k \leq d-1, \\
2^{k+1}-2 & \text { for } k=d\end{cases}\right.
$$

The value of $K=K_{0}+\cdots+K_{\min (k-1,0)}$ follows from the explicit form of the transformations in section 3

In Figure 6.2 we present the convergence history for the combinations CGL-GL and GJ-GL for $d=3$ and 4 and the integrand (6.2). As the approximate integral value 

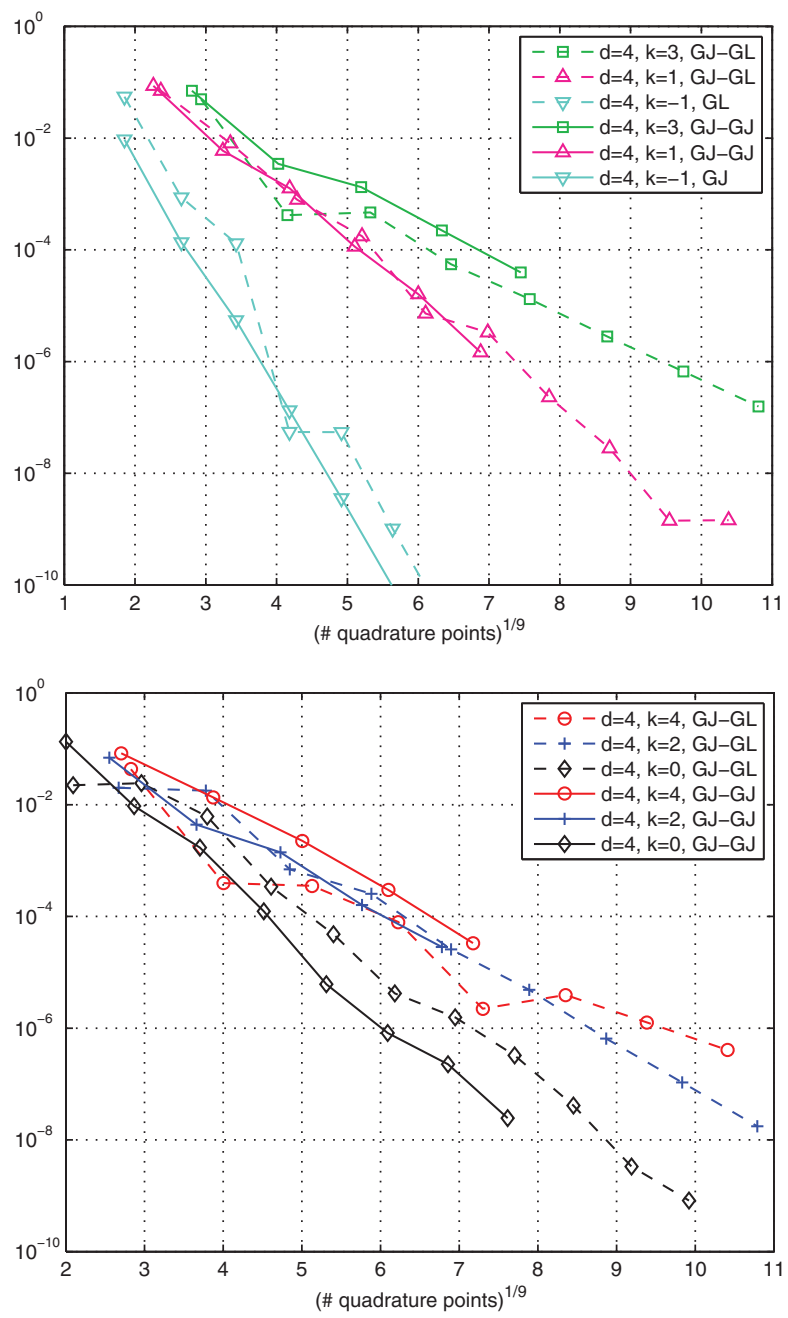

FIG. 6.3. Comparison of $G J$ and $G L$ in the regular direction for $F=\|y-x\|^{\alpha}$. Relative error $\left|I-Q_{N}\right| /|I|$ for $d=4$ and $k=-1, \ldots, d$.

we take the value of the GJ-GL quadrature on the finest discretization level. In both cases $d=3$ and $d=4$ we observe a strong improvement of several orders of magnitude if Gauss-Jacobi quadrature is used instead of the composite Gauss-Legendre rule in the singular direction.

In Figure 6.3 we compare the combinations GJ-GL and GJ-GJ. We observe that both quadrature rules provide almost the same quality of approximation: GJ-GJ is better for $k=-1,0$, GJ-GL is better for $k=3,4$, and the error curves are very close for $k=1,2$. Taking into account that the implementation of the Gauss-Jacobi quadrature rule in the regular direction is somewhat more complex (e.g., the weights of the input quadrature depend on the parameter $j$; cf. Remark 3 ) and the simplices with many common vertices (i.e., for high $k$ ) cause the largest quadrature error, the combination GJ-GL is advisable for implementation for problems with kernels satisfying (1.7). In the case of more general singularities (1.4) the combination CGLGL (not CGL-GJ) is advisable for the same reason. 
Appendix. Here we prove two auxiliary results on regularity of the product and composition of Gevrey- $\delta$ functions.

Proposition A.1. Suppose $f \in G^{\delta_{1}}(\Omega)$ and $g \in G^{\delta_{2}}(\Omega)$ for some $\Omega \subseteq \mathbb{R}^{m}$ and $\delta_{1}, \delta_{2} \geq 1$. Then the product $f g \in G^{\max \left\{\delta_{1}, \delta_{2}\right\}}(\Omega)$.

Proof. By definition (1.2), there exist $A_{0}, A_{1}, B_{0}, B_{1}>0$ such that

$$
\left|D^{\nu} f(x)\right| \leq A_{0} A_{1}^{|\nu|}(\nu !)^{\delta_{1}}, \quad\left|D^{\nu} g(x)\right| \leq B_{0} B_{1}^{|\nu|}(\nu !)^{\delta_{2}} \quad \forall \nu \in \mathbb{N}_{0}^{m}, \quad \forall x \in \Omega .
$$

Then, by the $m$-dimensional product rule

$$
\begin{aligned}
\left|D^{\nu} f g\right| & =\left|\sum_{0 \leq \beta \leq \nu}\left(\begin{array}{c}
\nu \\
\beta
\end{array}\right) D^{\nu-\beta} f D^{\beta} g\right| \\
& \leq \sum_{0 \leq \beta \leq \nu}\left(\begin{array}{c}
\nu \\
\beta
\end{array}\right) A_{0} A_{1}^{|\nu-\beta|}((\nu-\beta) !)^{\delta_{1}} B_{0} B_{1}^{|\beta|}(\beta !)^{\delta_{2}} \\
& \leq A_{0} B_{0}\left(A_{1}+B_{1}\right)^{|\nu|}(\nu !)^{\max \left\{\delta_{1}, \delta_{2}\right\}},
\end{aligned}
$$

and hence $f g \in G^{\max \left\{\delta_{1}, \delta_{2}\right\}}(\Omega)$ by (1.2).

Proposition A.2. Assume that $\delta_{1}, \delta_{2} \geq 1$ and $f \in G^{\delta_{1}}\left(\Omega_{1}\right), g \in G^{\delta_{2}}\left(\Omega_{2}\right)$ for some $\Omega_{2} \subseteq \mathbb{R}^{m_{2}}$ and Range $g \subseteq \Omega_{1} \subseteq \mathbb{R}^{m_{1}}$. Then $f \circ g \in G^{\delta_{1}+\delta_{2}-1}\left(\Omega_{2}\right)$.

Proof. We recall a multivariate version of the formula of Faà di Bruno [4], which represents the chain rule for composition of multivariate functions

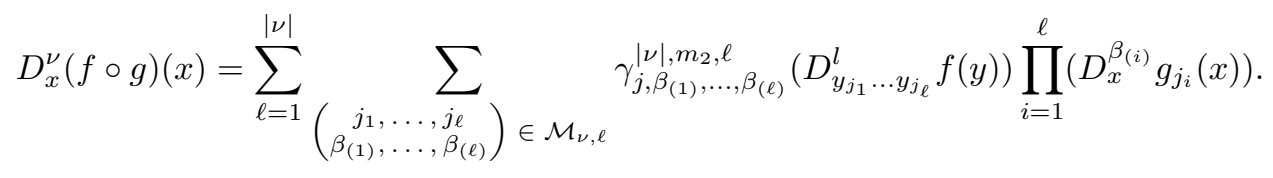

Here $\gamma_{j, \beta_{(1)}, \ldots, \beta_{(l)}}^{|\nu|, m_{2}, \ell}$ are positive integers, and the sum is taken over the set

$$
\mathcal{M}_{\nu, \ell}:=\left\{\left(\begin{array}{c}
j_{1}, \ldots, j_{\ell} \\
\beta_{(1)}, \ldots, \beta_{(\ell)}
\end{array}\right): \begin{array}{c}
j_{1}, \ldots, j_{\ell} \in\left\{1, \ldots, m_{1}\right\} \\
\beta_{(1)}+\cdots+\beta_{(\ell)}=\nu \wedge \beta_{(i)} \neq 0, i=1, \ldots, \ell
\end{array}\right\} .
$$

Note that $j_{1}, \ldots, j_{\ell}$ are integers and $\beta_{(1)}, \ldots, \beta_{(\ell)}$ are multi-indices of the same length as $\nu$, that is, $m_{2}$. We have

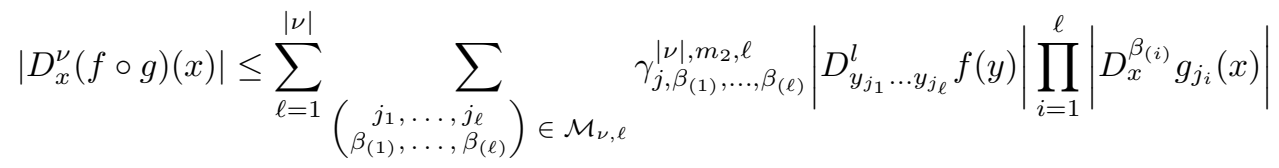

$$
\begin{aligned}
& \leq \sum_{\ell=1}^{|\nu|} \sum_{\left.\begin{array}{c}
j_{1}, \ldots, j_{\ell} \\
\beta_{(1)}, \ldots, \beta_{(\ell)}
\end{array}\right) \in \mathcal{M}_{\nu, \ell}} \gamma_{j, \beta_{(1)}, \ldots, \beta_{(\ell)}}^{|\nu|, m_{2}, \ell} A_{0} A_{1}^{\ell}(\ell !)^{\delta_{1}} \prod_{i=1}^{\ell} B_{0} B_{1}^{\left|\beta_{(i)}\right|}\left(\beta_{(i)} !\right)^{\delta_{2}} .
\end{aligned}
$$

From (A.1) we get $\sum_{i=1}^{\ell}\left|\beta_{(i)}\right|=|\nu|$ and $\prod_{i=1}^{\ell}\left(\beta_{(i)} !\right) \leq \nu !$; hence

$$
\begin{aligned}
\left|D_{x}^{\nu}(f \circ g)(x)\right| & \leq A_{0}\left(A_{1} B_{0} B_{1}\right)^{|\nu|} \sum_{\ell=1}^{|\nu|} \sum_{\left(\begin{array}{c}
j_{1}, \ldots, j_{\ell} \\
\beta_{(1)}, \ldots, \beta_{(\ell)}
\end{array}\right) \in \mathcal{M}_{\nu, \ell}} \gamma_{j, \beta_{(1)}, \ldots, \beta_{(\ell)}}^{|\nu|, m_{2}, \ell}(\ell !)^{\delta_{1}} \prod_{i=1}^{\ell}\left(\beta_{(i)} !\right)^{\delta_{2}} \\
& \leq A_{0}\left(A_{1} B_{0} B_{1}\right)^{|\nu|}(|\nu| !)^{\delta_{1}-1}(\nu !)^{\delta_{2}-1} \cdot \Theta
\end{aligned}
$$

Copyright (c) by SIAM. Unauthorized reproduction of this article is prohibited. 
with

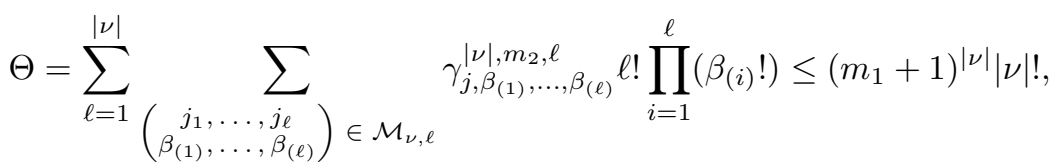

where in the last step we applied the identity [4, (1.24)]. The multinomial theorem yields $|\nu| ! \leq m_{2}{ }^{|\nu|} \nu !$; therefore,

$$
\left|D_{x}^{\nu}(f \circ g)(x)\right| \leq A_{0}\left(A_{1} B_{0} B_{1} m_{2}{ }^{\delta_{1}}\right)^{|\nu|}(\nu !)^{\delta_{1}+\delta_{2}-1} .
$$

Hence $f \circ g \in G^{\delta_{1}+\delta_{2}-1}\left(\Omega_{2}\right)$ by (1.2).

\section{REFERENCES}

[1] L. Boutet de Monvel And P. Krée, Pseudo-differential operators and Gevrey classes, Ann. Inst. Fourier (Grenoble), 17 (1967), pp. 295-323.

[2] A. Chernov, T. von Petersdorff, and C. Schwab, Quadrature Algorithms for Singular Integrals over High Dimensional Simplices, Technical report 1110, Institute for Numerical Simulation, University of Bonn, Bonn, Germany, 2011, in review.

[3] A. Chernov, T. von Petersdorff, and C. Schwab, Exponential convergence of hp quadrature for integral operators with Gevrey kernels, M2AN Math. Model. Numer. Anal., 45 (2011), pp. 387-422.

[4] M. Costabel, M. Dauge, And S. Nicaise, Corner Singularities and Analytic Regularity for Linear Elliptic Systems. Part I: Smooth Domains, HAL Archives, http://hal.archivesouvertes.fr/docs/00/45/41/17/PDF/CoDaNi_Analytic_Part_I.pdf, 2010.

[5] R. A. DeVore And L. R. Scott, Error bounds for Gaussian quadrature and weighted-L ${ }^{1}$ polynomial approximation, SIAM J. Numer. Anal., 21 (1984), pp. 400-412.

[6] L. Hörmander, The analysis of Linear Partial Differential Operators. III. Pseudodifferential Operators, Grundlehren Math. Wiss. 274, Springer-Verlag, Berlin, 1985.

[7] G. C. Hsiao and W. L. Wendland, Boundary Integral Equations, Appl. Math. Sci. 164, Springer-Verlag, Berlin, 2008.

[8] V. I. Krylov, Approximate Calculation of Integrals, Arthur H. Stroud, translator, Macmillan, New York, 1962.

[9] J.-C. NÉDÉLEC, Integral equations with nonintegrable kernels, Integral Equations Operator Theory, 5 (1982), pp. 562-572.

[10] S. Sauter and C. Schwab, Randelementmethoden, B. G. Teubner, Stuttgart, Germany, 2004.

[11] C. Schwaв, Variable order composite quadrature of singular and nearly singular integrals, Computing, 53 (1994), pp. 173-194.

[12] C. SChWAB AND W. L. Wendland, On numerical cubatures of singular surface integrals in boundary element methods, Numer. Math., 62 (1992), pp. 343-369.

[13] M. E. TAYLOR, Pseudodifferential operators, Princeton Math. Ser. 34, Princeton University Press, Princeton, NJ, 1981.

[14] L. N. Trefethen, Is Gauss quadrature better than Clenshaw-Curtis?, SIAM Rev., 50 (2008), pp. $67-87$.

Copyright $@$ by SIAM. Unauthorized reproduction of this article is prohibited. 